Institut für Medizinische Lehre und Ausbildungsforschung (Univ.-Prof. Dr. med. Sarah König, MME) der Medizinischen Fakultät der Universität Würzburg

\title{
Strukturiertes Feedback im Kommunikationstraining zur präoperativen Aufklärung: unterschiedliche Bewertungsquellen nutzbar machen
}

\author{
INAUGURAL-DISSERTATION \\ zur Erlangung des Doktorgrades \\ der Medizinischen Fakultät der \\ Julius-Maximilians-Universität zu Würzburg
}

vorgelegt von

Philipp Michael Kahr, B.A.

aus

Graz

Würzburg, März 2021 
Dekan: $\quad$ Univ.-Prof. Dr. med. Matthias Frosch

Referentin: Univ.-Prof. Dr. med. Sarah König, MME

Ko-Referentin: Univ.-Prof. Dr. med. Anne Simmenroth

Berichterstatterin:Univ.-Prof. Dr. rer. nat. Grit Hein

Datum der mündlichen Prüfung: 21. Juli 2021

Der Promovend ist Arzt. 


\section{Inhaltsverzeichnis}

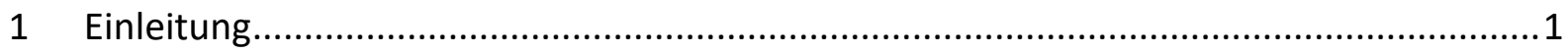

$1.1 \quad$ Feedback und Kommunikation im Medizinstudium ................................................

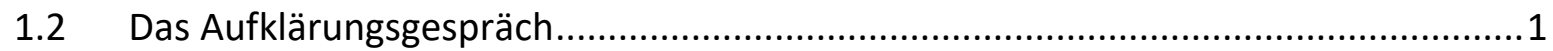

1.2.1 Der rechtliche Rahmen - Beteiligte, Inhalt, Zeit und Form.............................2

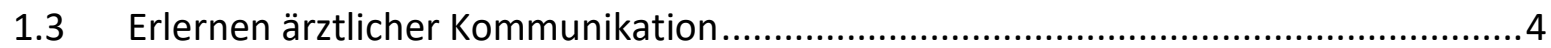

$1.4 \quad$ Neustrukturierung des Medizinstudiums .........................................................

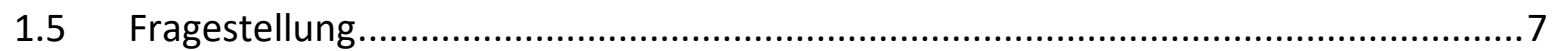

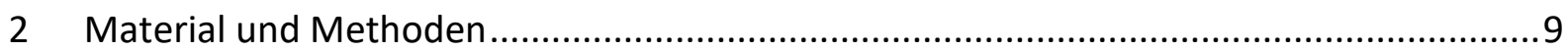

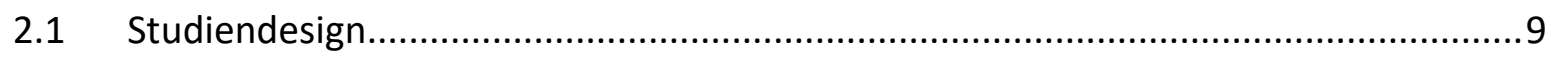

2.2 Selbststudium zur Vorbereitung auf das Kommunikationstraining ...........................

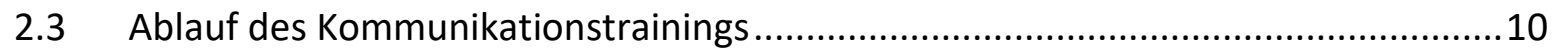

2.4 Checklisten als Grundlage für kompetentes Feedback ......................................14

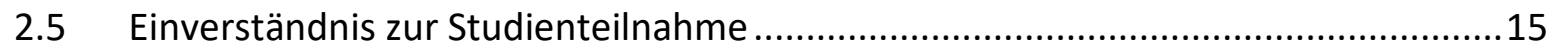

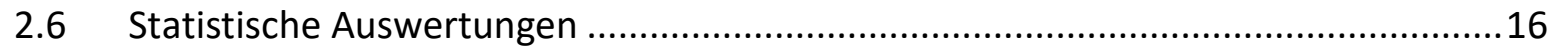

2.6.1 Testgüte der Bewertungschecklisten .......................................................16

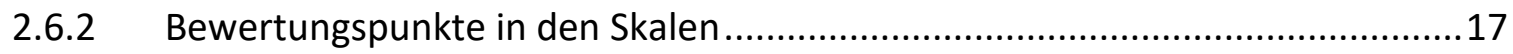

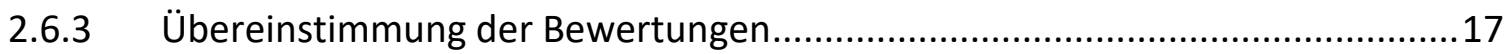

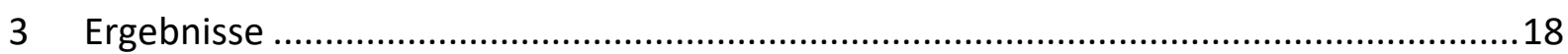

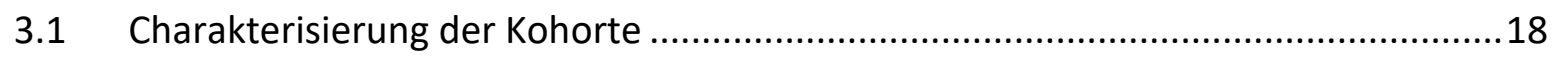

3.2 Qualität der Bewertungschecklisten............................................................... 18

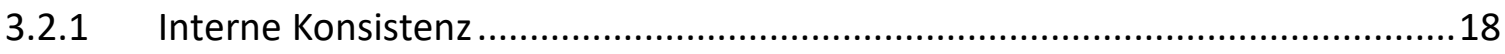

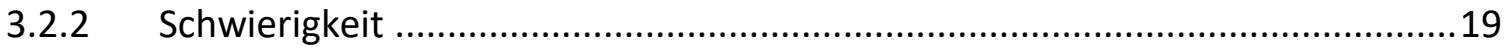

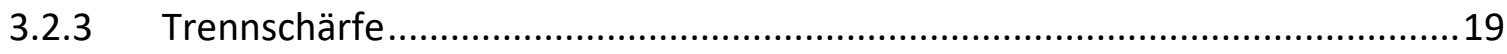


3.2.4 Tabellarische Übersicht der deskriptiven Itemkennwerte ..............................20

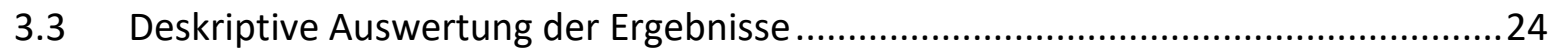

3.3.1 Punktescores in den Bewertungschecklisten nach Bewertungsquellen ............24

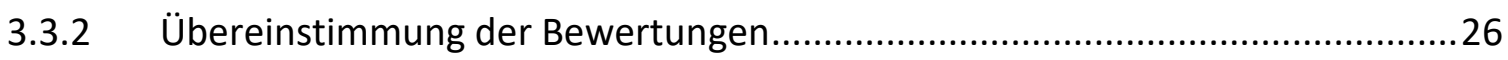

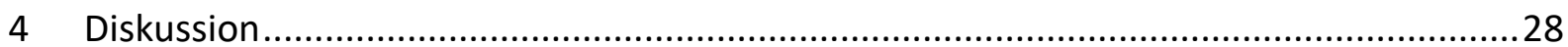

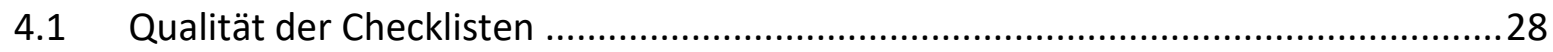

4.2 Analyse des strukturierten Feedbacks (360-Grad-Feedback) ................................30

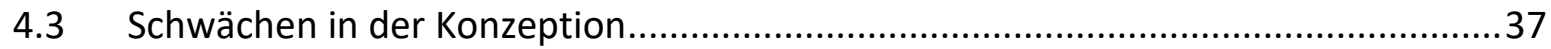

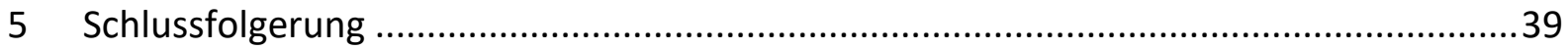

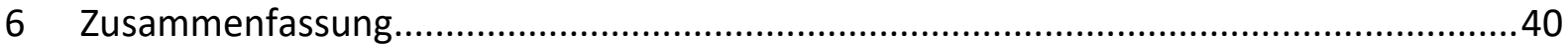

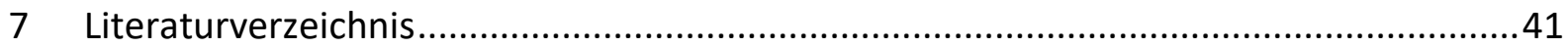

\section{Appendix}

I. Anhang A: Rollen der SP

II. Anhang B: Bewertungschecklisten

III. Abkürzungsverzeichnis

IV. Abbildungsverzeichnis

V. Tabellenverzeichnis

VI. Danksagung

VII. Lebenslauf

VIII. Eigene Veröffentlichung und Kongressteilnahme

IX. Ehrenwörtliche Erklärung 


\section{Einleitung}

\subsection{Feedback und Kommunikation im Medizinstudium}

Betreuung, Anleitung und Feedback spielen eine zentrale Rolle in der ärztlichen Ausund Weiterbildung [1]. Durch konstruktives Feedback wird sowohl die fachliche, als auch persönliche Entwicklung der Lernenden gefördert [2]. So bieten Feedbackgespräche nicht nur die Möglichkeit, Rückmeldungen zu den fachlichen Leistungen bzw. zum medizinischen Fachwissen oder zu Fertigkeiten zu geben, sondern auch die kommunikativen Kompetenzen in der Interaktion mit Patientinnen und Patienten oder im (interprofessionellen) Team zu beleuchten. Unbestritten ist, dass Kommunikationsfähigkeiten zu den wichtigen ärztlichen Kompetenzen zählen, die die Persönlichkeit und Wahrnehmung einer guten Ärztin/eines guten Arztes mitbestimmen [3].

Der im März 2017 verabschiedete Masterplan Medizinstudium 2020 legt ein besonderes Augenmerk auf die Kommunikation zwischen ärztlichem Personal und zu behandelnder Person und fordert die Vermittlung entsprechender Grundlagen möglichst früh im Studium [4].

\subsection{Das Aufklärungsgespräch}

Im chirurgischen Kontext lassen sich solche Fähigkeiten beispielsweise anhand des Aufklärungsgesprächs vor Operation trainieren. Zwischenmenschlich betrachtet bildet letzteres die Grundlage der Patientinnen- und Patienteninformation und schafft die Basis für ein ärztliches Vertrauensverhältnis. Im juristischen Kontext hat die präoperative Aufklärung insofern eine enorme Bedeutung, als jeder diagnostische oder therapeutische Eingriff in die körperliche Integrität der zu behandelnden Person per se eine Körperverletzung darstellt, die erst dadurch gerechtfertigt wird, dass die Person im Rahmen ihres Selbstbestimmungsrechts in den Eingriff einwilligt [5]. Die informiert und wohlüberlegt getroffene Entscheidung wird gemeinhin als „informed consent" bezeichnet. 


\subsubsection{Der rechtliche Rahmen - Beteiligte, Inhalt, Zeit und Form}

Neben der Durchführung der ärztlichen Behandlung stellt die Aufklärungspflicht eine Hauptpflicht der Ärztin/des Arztes aus dem Behandlungsvertrag mit der zu behandelnden Person dar. Gesetzlich ist diese Pflicht durch das im Februar 2013 in Kraft getretene Patientenrechtsgesetz im Bürgerlichen Gesetzbuch geregelt [6]. Im dortigen § 630c Abs. 2 ist folgendes festgelegt:

„Der Behandelnde ist verpflichtet, dem Patienten in verständlicher Weise zu Beginn der Behandlung und, soweit erforderlich, in deren Verlauf sämtliche für die Behandlung wesentlichen Umstände zu erläutern, insbesondere die Diagnose, die voraussichtliche gesundheitliche Entwicklung, die Therapie und die zu und nach der Therapie zu ergreifenden Maßnahmen. Sind für den Behandelnden Umstände erkennbar, die die Annahme eines Behandlungsfehlers begründen, hat er den Patienten über diese auf Nachfrage oder zur Abwendung gesundheitlicher Gefahren zu informieren. [...]"

Für die behandelnde Ärztin/den behandelnden Arzt ist eine korrekte und umfassend durchgeführte Aufklärung vor einem Eingriff von umso größerem Interesse, als diese/r die zu versorgende Person zwar mit dem Ziel eines Heilerfolgs behandelt, diesen aber nicht vertraglich garantieren kann. Eine solche Garantie kann die Ärztin/der Arzt auch allein schon deswegen nicht übernehmen, weil jede medizinische Behandlung ein natürliches Risiko in sich birgt. Den Beweis, dass ein Behandlungsfehler vorgelegen hat, muss im Klagefall die geschädigte Person erbringen. Allerdings liegt die Beweislast für eine ordnungsgemäße Aufklärung und die daraus resultierende Einwilligung auf Seiten des ärztlichen Personals [7].

Da das Gesetz ganz klar vom „Behandelnden“ spricht, ist das Aufklärungsgespräch von einer Ärztin/einem Arzt zu führen, die/der über die zur Durchführung des Eingriffs notwendige Ausbildung verfügt. Für die medizinische Aus- und Weiterbildung heißt das also, dass Studierende eine Aufklärung selbst dann nicht durchführen dürfen, wenn dies unter der Anwesenheit einer Ärztin/eines Arztes geschehen würde. Die Aufklärung durch eine nichtärztliche Person genügt den gesetzlichen Vorgaben schlichtweg nicht [6]. Gleichzeitig wird jedoch vom medizinischen Nachwuchs erwartet, dass er 
bereits mit dem Eintritt ins Berufsleben ein Aufklärungsgespräch durchführen kann. Dies erfordert in der Konsequenz ein geeignetes Training im Rahmen der Ausbildung.

Damit eine Aufklärung als vollständig gilt, muss sie die Diagnose, den Ablauf der vorgeschlagenen Behandlung sowie die damit einhergehenden Risiken und Komplikationen enthalten. In der klinischen Praxis ist zweifelsohne der letzte Punkt am bedeutendsten und mit den meisten Schwierigkeiten verbunden. Dabei reicht es bei der Risikoaufklärung, die Patientinnen/Patienten „im Großen und Ganzen“ aufzuklären - und zwar über vorübergehende oder dauerhafte Nebenfolgen des geplanten Eingriffs. Die Ärztin/der Arzt hat den Patientinnen/Patienten dabei zu vermitteln, wie wahrscheinlich und schwer eine mögliche Komplikation eintreten kann. Die Rate, mit welcher eine unerwünschte Nebenfolge auftreten kann, ist in diesem Zusammenhang nur von untergeordneter Bedeutung. Dies bedeutet, dass auch über seltene Komplikationen aufgeklärt werden muss, sofern diese besonders schwerwiegend sind und deswegen für die zu behandelnde Person von Interesse sein könnten, weil sie deren Lebensführung nachhaltig beeinträchtigen [8]. Dazu ein konkretes Beispiel aus unserem Kommunikationstraining: Eine durch die Studierenden aufzuklärende Operation war der offene Leistenhernienverschluss nach Lichtenstein. Bei diesem kann es bei männlichen Patienten zu einer Durchtrennung des Samenstrangs kommen. Da dies in der Praxis glücklicherweise sehr selten vorkommt, haben auch nicht alle Teilnehmenden über dieses Risiko aufgeklärt. Da unser besagter Schauspielpatient im Laufe der Lehrveranstaltung mehrere Gespräche durchlaufen hat, blieb dieses Risiko bei ihm hängen und er erwähnte bei den Feedbackgesprächen mit den Studierenden explizit, dass er dieses Risiko als Mann im zeugungsfähigen Alter als besonders schwerwiegend erachte und deswegen gerne darüber aufgeklärt werden wolle, auch wenn der Schadensfall in der Realität nur selten eintrete.

Ein häufiger Fehler im klinischen Alltag stellt eine nicht rechtzeitig durchgeführte Aufklärung dar. Doch welcher ist eigentlich der richtige Zeitpunkt für ein Aufklärungsgespräch? Generell gilt: Die Aufklärung hat stets vor dem ärztlichen Eingriff zu erfolgen. Mit Ausnahme von Notfällen oder sehr dringlichen Eingriffen muss der Zeitpunkt dabei 
so gewählt werden, dass den Patientinnen und Patienten noch genügend Zeit bleibt, das Für und Wider einer Behandlung abzuwägen und ihre Entscheidung zu überdenken. Dabei gilt nach allgemeiner Auffassung eine Aufklärung am Tag vor der Behandlung als rechtzeitig, wohingegen eine Aufklärung am Vorabend nach ständiger Rechtsprechung bereits als zu kurzfristig angesehen wird. Dies gilt für stationäre Eingriffe. Bei ambulanten Operationen muss wiederum unterschieden werden: Handelt es sich um kleinere, weniger komplikationsbehaftete Eingriffe, kann eine am Tag der Behandlung stattgefundene Aufklärung als rechtzeitig gelten, vorausgesetzt es besteht zwischen der Aufklärung und dem operativen Eingriff ein gewisser zeitlicher Abstand. Bei größeren oder komplizierteren Eingriffen ist eine Aufklärung am OP-Tag hingegen nie zeitgerecht [8].

Wurden die gesetzlichen Vorgaben hinsichtlich Inhalt und Zeitpunkt einer Aufklärung durch die ärztliche Person eingehalten, gilt es nun noch die richtige Form zu wahren. Dabei ist zu beachten, dass die präoperative Aufklärung stets mündlich erfolgen muss. Auch wenn im klinischen Alltag häufig standardisierte Merkblätter verwendet werden, so dürfen diese das ärztliche Aufklärungsgespräch nie ersetzen. Um im Falle eines Rechtsstreits die umfassende Aufklärung belegen zu können, ist außerdem eine adäquate Dokumentation unumgänglich. Sofern ein vorgefertigter Aufklärungsbogen verwendet wird, ist dieser durch zusätzliche (handschriftliche) Vermerke über Inhalt und Zeitpunkt des Gespräches zu individualisieren [8].

\subsection{Erlernen ärztlicher Kommunikation}

Mit Beginn des ärztlichen Schaffens muss jede Ärztin und jeder Arzt in der Lage sein, ein Aufklärungsgespräch durchzuführen. Aufgrund des rechtlichen Rahmens, welcher ein Aufklärungsgespräch in der klinischen Realität ausschließlich durch ärztliches Personal vorsieht, bedarf es praktischer Unterrichtseinheiten im geschützten und standardisierten Rahmen, z.B. mit Schauspielpatientinnen und -patienten (SP). Dies erfordert die Etablierung eines adäquaten Trainings auf studentischem Niveau. In ein solches Setting ist eine ausführliche Rückmeldung, sowohl über die kommunikativen Kompetenzen, als auch die chirurgischen Wissensaspekte hinsichtlich Operationstech- 
niken, Risiken bzw. Komplikationen des Eingriffs zu implementieren, damit sich für die Studierenden ein ernsthafter Lernfortschritt ergibt. Diesen gilt es sodann zu evaluieren. Auch der Nationale Kompetenzbasierte Lernzielkatalog Medizin (NKLM) betont die zentrale Bedeutung der Kommunikationsfähigkeit für den Arztberuf und die Gesundheitsversorgung. Neben einem allgemeinen Kapitel über „die Ärztin und den Arzt als Kommunikator" (Kapitel 7), findet sich dort auch ein eigener Abschnitt über die ärztliche Gesprächsführung (Kapitel 14c), in dem explizit betont wird, dass die Absolventin/der Absolvent der Medizin in der Lage sein müsse, ein Aufklärungsgespräch zu führen [9].

Gerade die Fähigkeiten in der Kommunikation zwischen behandelnder und behandelter Person lassen sich hervorragend mittels einer standardisierten, klinisch-praktischen Prüfung im Format der Objective Structured Clinical Examination (OSCE) [10] überprüfen, da diese zu einer Sensibilisierung für wesentliche Kompetenzen bereits im Studium führt [11].

Kiehl et al. haben 2014 die Leistungen von Studierenden bei einem Aufklärungstraining anhand von Videoaufnahmen der Gespräche von zwei unabhängigen Experten beurteilen lassen. Diese bewerteten zeitversetzt mittels Bewertungschecklisten die Studierenden, die die Ergebnisse im zeitlichen Abstand erhielten [12]. Eine Evaluation unter Medizinstudierenden der Universität Würzburg während des chirurgischen Blockpraktikums im Wintersemester 2016/17 ergab jedoch nach einem ähnlich konzeptionierten Training, dass ein kompetentes Feedback seitens einer ärztlichen Person mit Expertise und Praxiserfahrung zeitnah oder gar unmittelbar im Rahmen der Lehrveranstaltung gewünscht wird.

Dabei lassen sich gerade in Bezug auf praktische Übungen unter tutorieller Betreuung viele Vorteile ausmachen. Das konzeptionelle Element des sog. Peer-Assisted Learnings zielt darauf ab, dass sich die Studierenden gegenseitig beim Lernen unterstützen. Geschulte Studierende nehmen dabei im nicht-hierarchisch gestalteten Lehr-Lern-Kontext die Rolle der Lehrenden ein [13]. Das Konzept ist nicht neu und findet weltweilte Anwendung in der medizinischen Ausbildung [14]. Gerade, weil jede Ärztin/jeder Arzt in 
gewisser Hinsicht auch Lehrerin oder Lehrer ist (Rolle des Gelehrten im NKLM), sei es gegenüber den Patientinnen und Patienten, sei es gegenüber berufsjüngeren Kolleginnen und Kollegen, haben die medizinischen Universitäten die Verantwortung, die grundsätzliche Fähigkeit zu lehren, auch bereits im Studium zu vermitteln [15]. Im Hinblick auf das Kommunikationstraining zur Aufklärung vor OP bedeutet dies, dass Tutorinnen und Tutoren ihre Kommilitoninnen und Kommilitonen beobachten und dann eine ausführliche Rückmeldung geben, sowohl zu den kommunikativen Kompetenzen, als auch zum chirurgischen Wissensstand hinsichtlich Operationstechniken, Risiken bzw. Komplikationen des aufzuklärenden Eingriffs. Der niederschwellige und partizipative Unterrichtsstil ermöglicht dabei auch, die anderen Studierenden der Peer Group und auch die SP mit einzubeziehen. Im Rahmen eines 360-Grad-Feedbacks, bei dem unterschiedliche Bewertungsquellen berücksichtigt werden, kann schließlich das eigene Selbstbild mit unterschiedlichen Fremdbildern abgeglichen werden. Dadurch werden auch motivations- und leistungssteigernde Prozesse ermöglicht, welche die individuelle Entwicklung der Feedbackempfangenden unterstützen [16]. Gleichzeitig ergibt sich durch das didaktische Prinzip des Peer-Assisted Learnings jedoch auch für die Lehrenden ein messbarer Vorteil: So konnten Knobe et al. zeigen, dass studentische Tutorinnen/Tutoren, welche einen Kurs zur Befundung von Ultraschallbildern des Schultergelenks abhielten, in der darauffolgenden OSCE signifikant besser als die restlichen Studierenden abschnitten [17].

Um ein einheitliches Unterrichtskonzept zu ermöglichen, können Checklisten zur Strukturierung des Feedbacks dienen. Solche sind aus klinisch-praktischen Prüfungen, wie der OSCE, bekannt und lenken die Lernaktivitäten der Studierenden auf die zugrundliegenden Lernziele $[10,11]$. Wie verschiedene Studien gezeigt haben, stellen Bewertungschecklisten ein wirksames und zuverlässiges Instrument dar, um ein Feedback sinnvoll zu strukturieren und eine vollständige und zuverlässige Rückmeldung zu gewährleisten $[18,19]$. 


\subsection{Neustrukturierung des Medizinstudiums}

Betrachtet man den am 31. März 2017 verabschiedeten Masterplan 2020 zur Neustrukturierung des Medizinstudiums, so wurde darin auch ein Mustercurriculum Kommunikation als eine von 37 konkreten Maßnahmen implementiert. Im Detail findet sich dort folgende Formulierung (Maßnahme 8): „Anknüpfend an die Vorgaben der Approbationsordnung für Ärzte, die die ärztliche Gesprächsführung ausdrücklich als Gegenstand der ärztlichen Ausbildung und Inhalt der abschließenden Staatsprüfung vorgibt, und an die im NKLM hierzu entwickelten Lernziele, unterstützt das Bundesministerium für Gesundheit (BMG) den Erwerb kommunikativer Kompetenzen in der ärztlichen Ausbildung. Ziel ist, das Mustercurriculum „Nationales longitudinales Kommunikationscurriculum in der Medizin" in den Curricula der Hochschulen umzusetzen und spezielle Prüfungsformate hierfür zu entwickeln." Alleine durch diese Forderung ist mit einem enormen Aufwuchs an patientenbezogenen Unterrichtsstunden zu rechnen. In der Realität stellt der Gewinn von Ärztinnen und Ärzten für den Unterricht im Kleingruppenformat jedoch eine große organisatorische Herausforderung und gewichtige Personalressource dar. Deswegen können laut Empfehlung des Wissenschaftsrats nun erstmals auch Umsetzungsvarianten mit Online-Patientinnen und -Patienten, Patientenfällen in Seminaren oder auch Unterricht mit SP zählen [20]. Hieraus resultiert ein möglicher Mehrbedarf an studentischen Tutorinnen und Tutoren, die in Ergänzung zu ärztlichem Personal bzw. für den Vorteil kleinerer Gruppengrößen und Intensivierung der Übungsmöglichkeiten, den Unterricht mitbetreuen. Unberührt davon bleibt die ärztliche Ressource, die für den klassischen Unterricht am Krankenbett und für die klinischen Hospitationen bzw. für die arbeitsplatz-basierte Ausbildung vorgesehen ist und dort nicht ersetzt werden kann.

\subsection{Fragestellung}

Die vorliegende Studie adressierte die Frage, inwieweit es in einem Kommunikationstraining zur Aufklärung vor Operation die seitens der Studierenden gewünschten ärztlichen Expertinnen/Experten für die Rückmeldung der Aufklärungskompetenz braucht oder ob diese Rolle adäquat von geschulten Tutorinnen/Tutoren übernommen werden 
kann. Zur Klärung dieser Frage sollte auch auf die grundsätzliche Durchführbarkeit des Kommunikationstrainings mit studentischen Tutorinnen/Tutoren und SP eingegangen werden. Grundlage der Untersuchungen bildeten Bewertungschecklisten. Die Qualität dieser Checklisten sollte deshalb detailliert evaluiert werden, um deren Grundlage als Messinstrument zu rechtfertigen. Mit Blick auf die abgegebenen Bewertungen haben wir primär Bewertungsunterschiede zwischen den einzelnen Feedbackquellen untersucht und einzelne Items auf deren Beurteilerübereinstimmung geprüft.

Aufgezeigt werden sollte am Ende, wie sich der klinische Kommunikationsanlass eines präoperativen Aufklärungsgesprächs im Setting eines im Eigenstudium vorbereiteten und von studentischen Tutorinnen/Tutoren geleiteten Trainings üben und auswerten lässt. Darüber hinaus wurde die Frage nach der Qualität der unterschiedlichen Bewertungsquellen im Rahmen eines 360-Grad-Feedbacks beleuchtet, und zwar gemessen an einem Expertenfeedback. 


\section{Material und Methoden}

\subsection{Studiendesign}

Diese Querschnittsstudie wurde am Lehrstuhl für Medizinische Lehre und Ausbildungsforschung geplant und im Rahmen des Praktikums Chirurgie im Sommersemester 2017 durchgeführt. Es handelte sich dabei um eine Pflichtveranstaltung zur ärztlichen Aufklärung vor Operation für Humanmedizinstudierende des 8. Semesters. Jede/r Studierende nahm an nur einem von insgesamt 7 Terminen teil, die mittwochs für 90 Minuten in Seminarräumen der Lehrklinik abgehalten wurden. An jedem Termin fand ein Kommunikationstraining mit SP parallel in 3 bis 4 Kleingruppen statt. Jede der Gruppen wurde dabei von einer geschulten Tutorin/einem geschulten Tutor geleitet. Aus den Kleingruppen von jeweils 5-7 Studierenden wurden jeweils zwei „Aufklärende“ ausgelost, sodass über das gesamte Semester insgesamt 50 Aufklärungsgespräche stattfanden. Drei Szenarien wurden dabei nach dem Zufallsprinzip auf die Aufklärenden verteilt (siehe Tabelle 1).

Tabelle 1: Übersicht über die aufzuklärenden Eingriffe

\begin{tabular}{|l|l|}
\hline Krankheitsbild & chirurgischer Eingriff \\
\hline akute Appendizitis & laparoskopische Appendektomie \\
\hline Cholezystolithiasis & laparoskopische Cholezystektomie \\
\hline Leistenhernie & $\begin{array}{l}\text { offener Hernienverschluss mit Kunststoffnetz } \\
\text { (OP nach Lichtenstein) }\end{array}$ \\
\hline
\end{tabular}

\subsection{Selbststudium zur Vorbereitung auf das Kommunikationstraining}

Nach der Online-Anmeldung zu einem der sieben angebotenen Termine erfolgte die Vorbereitung im Vorfeld der Präsenztermine nach dem Flipped-Classroom-Konzept [21] mithilfe von Materialien auf der universitätseigenen e-Learning-Plattform WueCampus 2. Diese umfassten neben einem Podcast der Lehrstuhlinhaberin Univ.-Prof. Dr. med. Sarah König zu den Grundlagen der ärztlichen Aufklärung, den rechtlichen 
Aspekten sowie dem Ablauf eines Aufklärungsgesprächs auch die originalen proCompliance $^{\circledR}$-Aufklärungsbögen zu den drei Eingriffen, welche in der Klinik üblicherweise verwendet werden. Vom Georg Thieme Verlag in Stuttgart wurde außerdem ein Onlinekurs („Grundkurs zur Patientenaufklärung“) zu den rechtlichen Rahmenbedingungen der ärztlichen Aufklärung zur Verfügung gestellt.

Darüber hinaus fand sich in den Materialien auch die folgende Formulierung der Aufgabenstellung für das Kommunikationstraining:

Sie sind Assistenzärztin/Assistenzarzt auf einer chirurgischen Station. Führen Sie das Aufklärungsgespräch im Hinblick auf die bevorstehende Operation mit der/dem SP durch. Erklären Sie zunächst kurz, weshalb operiert werden muss und welches OPVerfahren empfohlen wird. Erläutern Sie dann die allgemeinen und speziellen Operationsrisiken und gehen Sie abschließend auf die postoperativen Verhaltensmaßnahmen ein. Versuchen Sie sich im Gesprächsverlauf an den Bedürfnissen der Patientin/des Patienten zu orientieren und ermöglichen Sie Raum für Fragen.

\subsection{Ablauf des Kommunikationstrainings}

An jedem Termin fanden sich vier Kleingruppen (A, B, C und D) um 15:00 Uhr in der Lehrklinik (Josef-Schneider-Str. 2, Haus D5) zusammen. Jede Gruppe führte Aufklärungsgespräche mit zwei SP, dazu waren jeweils in Folge zwei Sitzungen von je $45 \mathrm{Mi}$ nuten vorgesehen. Die/Der SP teilte den Studierenden die bevorstehende Operation (Appendektomie, Cholezystektomie, Leistenhernienverschluss) mit. Die aufklärende Person wurde aus der Gruppe per Los gezogen und führte das Gespräch durch (maximal 10 Minuten). Die anderen fünf Studierenden beobachteten das Gespräch, welches mit einer Kamera auf eine Speicherkarte aufgenommen wurde. Nach der OPAufklärung bewerteten alle Teilnehmenden das Gespräch mit Hilfe einer FeedbackCheckliste und bereiteten die Rückmeldung vor. In dieser Zeit schaute sich die/der Aufklärer/in das Video in einem Nebenraum auf einem iPad an und bewertete sich selbst (Dauer 10 Minuten). Anschließend kam sie/er zurück zur Gruppe und erhielt eine mündliche Rückmeldung im Rahmen eines sog. 360-Grad-Feedbacks von der/dem 
$\mathrm{SP}$, den Kommilitoninnen und Kommilitonen und der/dem Tutor/in. Unklare Aspekte wurden abschließend gemeinsam diskutiert.

Zur zweiten Sitzung wurden danach die Räume so getauscht, dass jede Gruppe eine/n andere/n SP mit anderer Erkrankung bekam. Dann wurde wieder gelost (nur unter den beobachtenden Mitstudierenden des ersten Durchlaufs) und die gesamte Prozedur mit einer anderen Operation wiederholt (erneut 45 Minuten).

Zusätzlich wurden, sofern die Aufklärenden ihr Einverständnis gegeben hatten, die Videos am Ende des Semesters von einem Facharzt für Chirurgie mit Erfahrung in OSCE-Prüfungen (Experte) bewertet. Das Ergebnis dieser Referenzbewertung wurde den Studierenden auf Wunsch mitgeteilt. Am Ende lagen den Studierenden also Bewertungen aus folgenden Quellen vor: aufklärende Person (= Selbstbewertung), Peers, SP, Tutor/in und Experte. Eine detaillierte Darstellung des zeitlichen Ablaufs eines Kommunikationstrainings findet sich in Tabelle 2. 


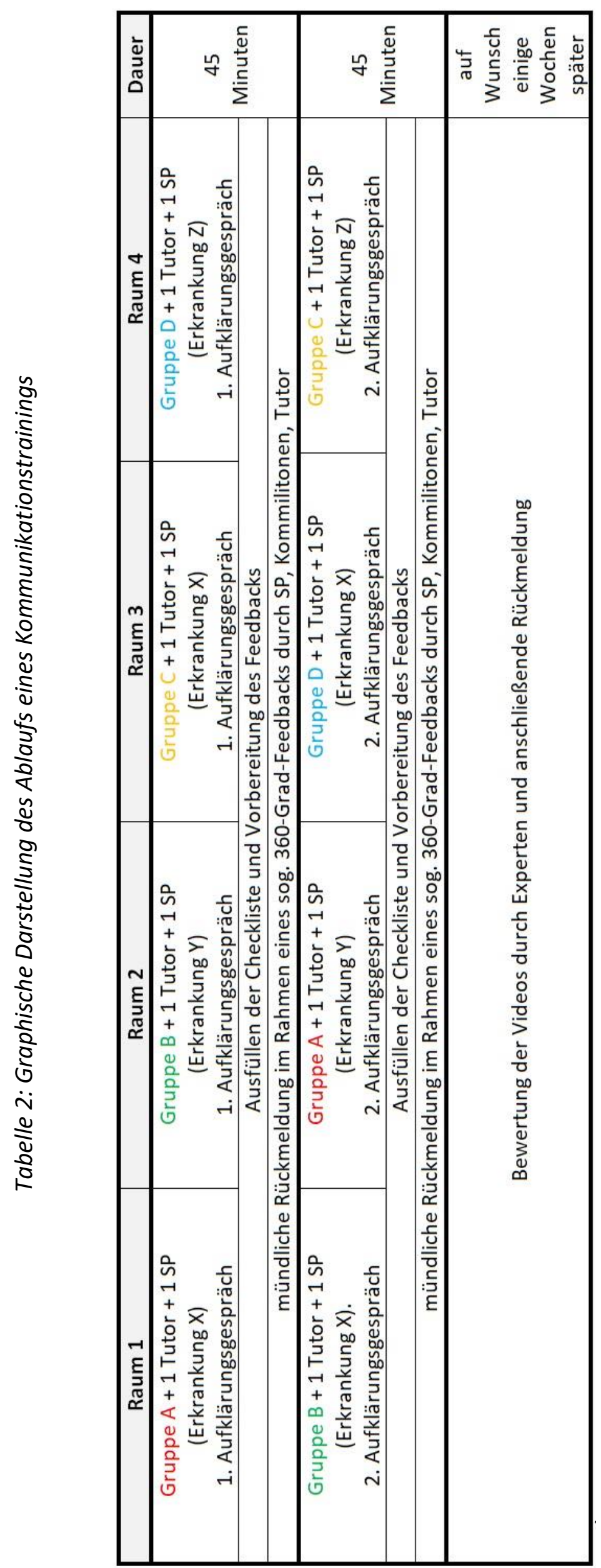


Jede Studierendengruppe wurde von einer/einem hinsichtlich der chirurgischen Inhalte und kommunikativen Kompetenzen geschulten, studentischen Übungsleitenden (Tutor/in) geleitet. Diese/r sollte neben der Moderation der Abläufe zusätzlich zu den ebenfalls geschulten SP sowie den Peers ein kompetentes Feedback zur Leistung der Aufklärenden geben. Die Schulung der Feedbackgebenden erfolgte nach dem „Frame of Reference Training", um eine möglichst hohe Beobachterübereinstimmung sowie eine einheitliche Beurteilung nach einem gemeinsam festgelegten Bezugsrahmen zu erreichen [22]. Die SP erhielten eine separate Schulung nach den vorgenannten Standards sowie eine Erklärung ihrer Rolleneigenschaften. Die SP wurden hierbei angewiesen, den Studierenden zugewandt und interessiert entgegenzutreten, ohne jedoch die Aufklärenden durch skurriles oder emotionales Verhalten zu überfordern. Zusätzlich waren die SP angehalten auf die Erwähnung bestimmter Aspekte zu achten und konkret nachzufragen, falls diese nicht bereits ungefragt von den Aufklärenden erwähnt wurden. Unterbrechen sollten die SP die Aufklärenden nur dann, wenn sie etwas nicht verstanden haben. Die drei möglichen Rollen der SP waren wie folgt definiert:

- Frau/Herr Körte: Vorstellung zur laparoskopischen Appendektomie bei am Morgen aufgetretenen starken Unterbauschmerzen und zweimaligem Erbrechen. Die OP sollte in den nächsten 2-3 Stunden stattfinden. Es bestehen keine relevanten Vorerkrankungen.

- Frau/Herr Weber: Vorstellung zur elektiven laparoskopischen Cholezystektomie bei seit längerer Zeit bestehendem Gallensteinleiden bei Z.n. vor 6 Wochen durchgeführter ERCP. Es wird keine Dauermedikation eingenommen.

- Frau/Herr Bäcker: Vorstellung zum offenen Leistenhernienverschluss mit Netzimplantation (OP nach Lichtenstein) bei schmerzhafter Vorwölbung in der linken Leiste. Da der Bruch aktuell nicht eingeklemmt ist, findet die OP geplant und nicht als Notfalleingriff statt. An Vorerkrankungen bestehen ein Asthma bronchiale sowie diverse Allergien.

Die ausführlichen Rollenbeschreibungen befinden sich im Anhang A. 


\subsection{Checklisten als Grundlage für kompetentes Feedback}

Für die standardisierte Bewertung wurden für alle drei Operationen bzw. Aufklärungsgespräche eigene Bewertungschecklisten (basierend auf jenen von Kiehl et al. [12]) entwickelt und mit der Software der Electric Paper Evaluationssysteme GmbH in Lüneburg („EvaSys“) erstellt. Im ersten Teil wurden allgemeine Angaben wie Gruppe, Eingriff und Termin erfasst, womit eine Zuordnung zu den Videoaufzeichnungen der Aufklärungsgespräche ohne Nennung von Namen bzw. Personen gewährleistet war. Der zweite Teil war für jede der drei aufzuklärenden Operationen identisch und ermöglichte eine Beurteilung der allgemeinen Fähigkeiten zur Kommunikation und Interaktion mit den SP. Hierbei handelte es sich um die Skala „Kommunikation“ mit 11 Items auf einer dreistufigen Likert-Skala (1 Punkt für „nicht erfüllt“ bis 3 Punkte für „voll erfüllt“). Die Konzeption erfolgte auf Basis des Calgary Cambridge Guides [23], aufgrund der Aufklärungssituation wurden die Items der Skala entsprechend modifiziert. Im dritten Teil der Checklisten wurde die Nennung der einzelnen OP-Risiken und Komplikationen (Skala „Komplikationen“) erhoben. Hierzu mussten die Bewertungschecklisten spezifisch geschrieben werden: mit 17 Items (Appendektomie), 20 Items (Cholezystektomie) bzw. 19 Items (Leistenhernie). Die Vergabe der vollen Punktzahl („voll erfüllt“) wurde restriktiv gehandhabt und mit entsprechenden Hinweisen in den Schulungen auf das Erfordernis hingewiesen.

In der folgenden Abbildung 1 ist ein Beispiel des zweiten Teils der Bewertungschecklisten, welcher für alle drei Eingriffe identisch war, zu sehen. Die vollständigen Checklisten finden sich unter Anhang B dieser Arbeit. 


\section{Kommunikative Kompetenz und Interaktion mit Schauspielpatient}

2.1 ... stellt sich mit Namen vor.

2.2 ... fragt nach dem aktuellen Befinden.

2.3 ... tritt dem Patienten wertschätzend gegenüber.

2.4 ... hat einen "roten Faden" in der Gesprächsführung.

$2.5 \ldots$ verwendet ein angepasstes Sprechtempo.

2.6 .... verwendet verständliche Formulierungen.

2.7 ... benutzt den Aufklärungsbogen zur

Veranschaulichung des Eingriffs.

2.8 ... hat eine dem Patienten zugewandte Körperhaltung.

2.9 ... hält Blickkontakt.

$2.10 \ldots$ greift möglicherweise geäußerte Ängste des Patienten auf.

2.11 ... gibt mehrfach Gelegenheit für Nachfragen.

\section{Abbildung 1: Auszug aus einer Bewertungscheckliste}

\subsection{Einverständnis zur Studienteilnahme}

Die Teilnahme an der Studie war für die Studierenden freiwillig. Für die Aufzeichnung der Aufklärungsgespräche mittels einer digitalen Videokamera auf Speicherkarten wurde von allen teilnehmenden Studierenden und den SP eine schriftliche Einverständniserklärung eingeholt. 45 Studierende (90\% aller aufklärenden Personen) stimmten einer Aufzeichnung ihrer Gespräche zu. Das Ausfüllen der Bewertungschecklisten erfolgte anonym unter Kennzeichnung der Bewertungsquelle. Eine Beratungspflicht gemäß § 15 Berufsordnung der Ärztinnen und Ärzte lag nicht vor, da die vorliegende Studie keine biomedizinische Forschung am Menschen beinhaltete. Die datenschutzrechtlichen Bestimmungen gemäß Bayerischem Datenschutzgesetz wurden eingehalten. So wurden sämtliche Daten ausschließlich in anonymisierter Form erhoben und von Frau Dipl.-Psych. Sonia Sippel sowie dem Autor dieser Arbeit auf Rechnern des Instituts für Medizinische Lehre und Ausbildungsforschung des Universitätsklinikums Würzburg verarbeitet. Die handschriftlich ausgefüllten Bewertungschecklisten wurden nach deren digitaler Erfassung vernichtet. Die Videoaufzeichnungen der Aufklärungsgespräche wurden ebenso auf einem Rechner des Instituts archiviert, wo sie vor unberechtigtem Zugriff geschützt sind. 


\subsection{Statistische Auswertungen}

Im Rahmen der Studie wurden 442 Bewertungschecklisten von 180 Personen (171 Studierende, 4 Tutorinnen/Tutoren, 4 SP, 1 Experte) zu drei verschiedenen Operationen (Appendektomie $n=186$; Cholezystektomie $n=121$; Leistenhernie $n=135$ ) ausgefüllt. Die Bewertungschecklisten wurden eingescannt, die Bewertungen somit digital erfasst und dann die Daten mit der Software SPSS 24 statistisch ausgewertet. Die statistische Auswertung hat eine Mitarbeiterin des Instituts für Medizinische Lehre und Ausbildungsforschung des Universitätsklinikums Würzburg, Frau Dipl.-Psych. Sonia Sippel, durchgeführt. Mit der Berechnung von Cronbachs Alpha, Schwierigkeit und Trennschärfe wurde die Güte der Bewertungschecklisten untersucht. Die deskriptive Auswertung umfasste Häufigkeiten, Mittelwerte und Standardabweichungen aller Items der Bewertungschecklisten. Darüber hinaus wurden Übereinstimmungen zwischen den Bewertenden mithilfe des Intraklassenkorrelationskoeffizienten (ICC) [24] ermittelt und Mittelwertunterschiede auf ihre Signifikanz hin überprüft.

\subsubsection{Testgüte der Bewertungschecklisten}

Cronbachs Alpha wird als Kennzahl für die interne Konsistenz der Items angesehen und zeigt auf, inwieweit die einzelnen Items miteinander korrelieren [25]. Der Wertebereich liegt zwischen 0 und 1 und sollte entsprechend weiten Teilen der Literatur bei mindestens 0,7 liegen, wenngleich es keinen objektiven Grenzwert gibt, ab welchem man eine Skala verwenden darf [26].

Die Schwierigkeit eines Items ist definiert als die mittlere bei diesem Item erreichte Punktzahl. Die maximal erreichbare Punktzahl pro Item lag jeweils bei 3, dies entsprach also 100 \%. Die Schwierigkeit (mittlere erreichte Punktzahl bezogen auf die maximal erreichbare Punktzahl) sollte entsprechend den in der Literatur angegebenen Referenzwerten zwischen 40 und 80 \% liegen, in unserem Fall also zwischen einem Wert von 1,8 und 2,6 Punkten [27]. Je näher der Wert bei 3,0 lag, desto mehr Studierende haben das jeweilige Item „voll erfüllt“, was für einen niedrigeren Schwierigkeitsgrad des Items sprach. Umgekehrt wies eine niedrige erreichte Punktezahl im Mittel auf eher schwierige Items hin. 
Die Trennschärfe ermöglicht eine Aussage darüber, inwieweit ein einzelnes Item mit dem Gesamtergebnis des Tests korreliert und ist geeignet zwischen guten und schlechten Aufklärenden zu unterscheiden. Diese kann Werte zwischen -1 und 1 einnehmen, wobei ein negativer Wert aufzeigen kann, dass das Item umgekehrt wie beabsichtigt verstanden wurde. Trennschärfen um Null weisen darauf hin, dass das Item keinen inhaltlichen Zusammenhang mit den restlichen Items aufweist. Ein hoher positiver Wert drückt aus, dass das einzelne Item etwas Ähnliches wie der Gesamttest misst [28]. Ein Wert von 0,2 wird in der gängigen Literatur allgemein als Schwelle genannt wird, ab der ein Item als akzeptabel gilt [27].

\subsubsection{Bewertungspunkte in den Skalen}

Unterschiede zwischen den Bewertungen innerhalb der einzelnen Skalen (Kommunikation bzw. Risiken) wurden mithilfe einer Varianzanalyse (ANOVA) untersucht und durch einen Post-Hoc-Test ergänzt. Zur Anwendung kam der Scheffé-Test, welcher alle Feedbackgebende paarweise miteinander verglich [29]. Mit der Bonferroni-Korrektur wurde eine fehlerhafte Anhäufung von signifikanten Unterschieden zwischen den Bewertungen statistisch vermieden [29].

Wo ein signifikanter Unterschied zwischen den Mittelwerten der Bewertungen bestand, wurde zusätzlich Cohens d als Maß für die Effektstärke berechnet, um zu ermitteln, ob die signifikanten Unterschiede eine praktische Relevanz haben [30].

\subsection{3 Übereinstimmung der Bewertungen}

Um die Beurteilerübereinstimmung quantitativ aufzuzeigen, wurden Intraklassenkorrelationskoeffizienten (ICC) errechnet, da diese ein geeignetes Maß für intervallskalierte Ratings darstellen. Dabei wurde für alle Bewertungschecklisten mit ihren beiden Skalen der unjustierte ICC herangezogen, da dieser in der Literatur als strenger klassifiziert wird [24]. 


\section{Ergebnisse}

\subsection{Charakterisierung der Kohorte}

An der Studie haben insgesamt 180 Personen teilgenommen. Diese setzten sich zusammen aus 100 Studentinnen und 71 Studenten im 8. Fachsemester ihres Studiums der Humanmedizin. Diese wurde von 2 Tutorinnen und 2 Tutoren angeleitet, die ebenfalls demselben Semester angehörten. Hinzu kamen 4 Simulationspersonen, welche die Patientinnen bzw. Patienten spielten und ein Facharzt für Viszeralchirurgie, welcher als Experte fungierte und dessen Referenzbewertung als zuverlässiges Außenkriterium in der vorliegenden Studie herangezogen wurde.

\subsection{Qualität der Bewertungschecklisten}

Als Grundlage für das aus mehreren Bewertungsquellen bestehende Feedback im Kommunikationstraining dienten drei den aufzuklärenden Eingriffen angepasste Bewertungschecklisten. Um beurteilen zu können, inwieweit die Checklisten in die Analyse der Bewertungsquellen miteinbezogen werden können, wurden verschiedene Maßzahlen berechnet. Betrachtet wurden dabei die interne Konsistenz der Items innerhalb einer Checkliste, also Cronbachs Alpha, sowie die Schwierigkeit einzelner Items und die Korrelation einzelner Items mit dem Gesamtergebnis des Tests, also die sogenannte Trennschärfe.

\subsubsection{Interne Konsistenz}

Als Maßzahl für die interne Konsistenz wurde Cronbachs Alpha für alle drei Bewertungschecklisten mit ihren beiden Skalen („Kommunikation“ und „Komplikationen“) berechnet. Die Werte reichten von 0,52 bis 0,71 . Dabei fiel der Wert für die Bewertungscheckliste „Leistenhernie“ mit 0,71 (für beide Skalen) am höchsten bzw. verlässlichsten aus. Eine Übersicht über diese Werte findet sich in Tabelle 3 [31]. 
Tabelle 3: Cronbachs Alpha für die 3 Bewertungschecklisten (Anzahl der Items je Skala in Klammern)

\begin{tabular}{|l|c|c|}
\hline \multicolumn{1}{|c|}{ Bewertungscheckliste } & Skala & Cronbachs Alpha \\
\hline Appendektomie & Kommunikation (11) & 0,68 \\
\hline & Komplikationen (17) & 0,57 \\
\hline Cholezystektomie & Kommunikation (11) & 0,52 \\
\hline & Komplikationen (20) & 0,61 \\
\hline Leistenhernie & Kommunikation (11) & 0,71 \\
\hline & Komplikationen (19) & 0,71 \\
\hline
\end{tabular}

\subsubsection{Schwierigkeit}

Die Schwierigkeit (mittlere erreichte Punktzahl bezogen auf die maximal erreichbare Punktzahl) sollte zwischen 40 \% und 80 \% liegen, in unserem Fall also zwischen 1,8 und 2,6 Punkten [27]. Auffällig war, dass bei allen drei Bewertungschecklisten in der Skala „Kommunikation“ die meisten Items oberhalb dieses Referenzbereiches lagen, also eher sehr leichten Items entsprachen. In der Skala „Komplikationen“ zeigten in der Checkliste „Appendektomie“ 14 von 17 Items eine erwünschte Schwierigkeit, in der „Cholezystektomie“ war es die Hälfte der Items, bei der „Leistenhernie“ lediglich 6 von 19 Items. Die durchschnittlichen Schwierigkeiten waren jedoch vergleichbar: „Appendektomie“ mit 2,41, „Cholezystektomie“ mit 2,20 und „Leistenhernie“ mit 2,22 Punkten. Somit konnte gerechtfertigt werden, dass die Studierenden in den unterschiedlichen Operationsszenarien unterrichtet bzw. bewertet wurden.

\subsubsection{Trennschärfe}

Generell zeigte sich, dass die Mehrheit der Items über alle drei Checklisten hinweg eine Trennschärfe von über 0,2 aufwies. Damit zeigten die Checklisten eine akzeptable mittlere Trennschärfe, was für die weitere Auswertung über alle drei Checklisten sprach. Interessant erscheint dabei, dass jene Items, welche die Kommunikation betreffen, insgesamt stärker mit der Gesamtnote korrelieren als die restlichen. 


\subsubsection{Tabellarische Übersicht der deskriptiven Itemkennwerte}

Eine detaillierte Aufstellung über die erreichten Mittelwerte als Ausdruck der Schwierigkeit sowie die Trennschärfe aller Items in den unterschiedlichen Bewertungschecklisten liefern die folgenden Tabellen 4-6. Für das erste Item der Skala „Kommunikation", "stellt sich mit Namen vor", konnte keine sinnvolle Trennschärfe berechnet werden, da dieses Item von allen Studierenden erfüllt wurde.

Tabelle 4: Deskriptive Itemkennwerte zur Bewertungscheckliste "Appendektomie“

$M=$ Mittelwert $; S D=$ Standardabweichung; Min = niedrigster erreichter Wert; Max = höchster erreichter Wert; \% = Schwierigkeit in Prozent; $S=$ Trennschärfe; $C A D=$ Cronbachs Alpha, wenn Item weggelassen

\begin{tabular}{|c|c|c|c|c|c|c|c|}
\hline Item & M & SD & Min & Max & $\%$ & $\mathbf{S}$ & CAD \\
\hline \multicolumn{8}{|c|}{ Kommunikation } \\
\hline stellt sich mit Namen vor. & 3,00 & 0,00 & 3,00 & 3,00 & 100,00 & & \\
\hline $\begin{array}{l}\text { fragt nach dem aktuellen Befin- } \\
\text { den. }\end{array}$ & 2,54 & 0,80 & 1,00 & 3,00 & 77,08 & 0,32 & 0,67 \\
\hline $\begin{array}{l}\text { tritt den Patientinnen und Pati- } \\
\text { enten wertschätzend gegen- } \\
\text { über. }\end{array}$ & 2,89 & 0,31 & 2,00 & 3,00 & 94,71 & 0,24 & 0,67 \\
\hline $\begin{array}{l}\text { hat einen 'roten Faden' in der } \\
\text { Gesprächsführung. }\end{array}$ & 2,70 & 0,46 & 2,00 & 3,00 & 85,14 & 0,43 & 0,64 \\
\hline $\begin{array}{l}\text { verwendet ein angepasstes } \\
\text { Sprechtempo. }\end{array}$ & 2,74 & 0,46 & 1,00 & 3,00 & 87,24 & 0,30 & 0,66 \\
\hline $\begin{array}{l}\text { verwendet verständliche For- } \\
\text { mulierungen. }\end{array}$ & 2,73 & 0,46 & 1,00 & 3,00 & 86,63 & 0,45 & 0,64 \\
\hline $\begin{array}{l}\text { benutzt den Aufklärungsbogen } \\
\text { zur Veranschaulichung des Ein- } \\
\text { griffs. }\end{array}$ & 2,81 & 0,49 & 1,00 & 3,00 & 90,48 & 0,38 & 0,65 \\
\hline $\begin{array}{l}\text { hat eine den Patientinnen und } \\
\text { Patienten zugewandte Körper- } \\
\text { haltung. }\end{array}$ & 2,93 & 0,28 & 1,00 & 3,00 & 96,41 & 0,26 & 0,67 \\
\hline hält Blickkontakt. & 2,81 & 0,39 & 2,00 & 3,00 & 90,58 & 0,32 & 0,66 \\
\hline $\begin{array}{l}\text { greift möglicherweise geäußer- } \\
\text { te Ängste der Patientinnen und } \\
\text { Patienten auf. }\end{array}$ & 2,43 & 0,68 & 1,00 & 3,00 & 71,43 & 0,40 & 0,64 \\
\hline $\begin{array}{l}\text { gibt mehrfach Gelegenheit für } \\
\text { Nachfragen. }\end{array}$ & 2,53 & 0,63 & 1,00 & 3,00 & 76,44 & 0,39 & 0,64 \\
\hline \multicolumn{8}{|c|}{ Komplikationen } \\
\hline Allergie/Unverträglichkeit & 2,81 & 0,54 & 1,00 & 3,00 & 90,69 & 0,02 & 0,57 \\
\hline Lagerungsschäden & 1,63 & 0,89 & 1,00 & 3,00 & 31,71 & $-0,02$ & 0,59 \\
\hline Gewebeschäden durch Strom & 1,74 & 0,91 & 1,00 & 3,00 & 37,03 & 0,12 & 0,56 \\
\hline Verletzung von Nerven im Ope- & 2,56 & 0,73 & 1,00 & 3,00 & 78,02 & 0,24 & 0,54 \\
\hline
\end{tabular}




\begin{tabular}{|l|c|c|c|c|c|c|c|}
\hline rationsgebiet & & & & & & & \\
\hline $\begin{array}{l}\text { (Nach-)Blutung, Blutübertra- } \\
\text { gung, Infektionsrisiko }\end{array}$ & 2,54 & 0,63 & 1,00 & 3,00 & 77,15 & 0,38 & 0,52 \\
\hline $\begin{array}{l}\text { Verletzung von Nachbarorga- } \\
\text { nen (Darm, Harnblase, innere } \\
\text { Genitale) }\end{array}$ & 2,49 & 0,67 & 1,00 & 3,00 & 74,47 & 0,21 & 0,55 \\
\hline $\begin{array}{l}\text { Verletzung von Gefäßen, insb. } \\
\text { beim Setzen der Trokare }\end{array}$ & 2,01 & 0,80 & 1,00 & 3,00 & 50,28 & 0,23 & 0,54 \\
\hline $\begin{array}{l}\text { Hautemphysem (durch Reste } \\
\text { des Kohlendioxids) }\end{array}$ & 2,51 & 0,78 & 1,00 & 3,00 & 75,54 & 0,25 & 0,54 \\
\hline Schulter-/Bauchschmerzen & 1,88 & 0,90 & 1,00 & 3,00 & 44,02 & 0,08 & 0,57 \\
\hline Pneumothorax, Gasembolie & 1,85 & 0,91 & 1,00 & 3,00 & 42,58 & 0,25 & 0,54 \\
\hline $\begin{array}{l}\text { Übersäuerung/Azidose } \\
\text { Thrombose, Embolie }\end{array}$ & 2,33 & 0,89 & 1,00 & 3,00 & 66,67 & 0,06 & 0,58 \\
\hline $\begin{array}{l}\text { Wundinfektion, Abszess im } \\
\text { Abdomen, Peritonitis }\end{array}$ & 2,32 & 0,80 & 1,00 & 3,00 & 73,78 & 0,25 & 0,54 \\
\hline Nahtinsuffizienz, Stuhlfisteln & 2,06 & 0,73 & 1,00 & 3,00 & 66,03 & 0,29 & 0,54 \\
\hline Platzbauch, Narbenbruch & 2,00 & 0,86 & 1,00 & 3,00 & 50,00 & 0,25 & 0,54 \\
\hline $\begin{array}{l}\text { Narbenbildung, kosmetisch } \\
\text { ungünstiges Ergebnis }\end{array}$ & 2,29 & 0,75 & 1,00 & 3,00 & 64,48 & 0,27 & 0,54 \\
\hline $\begin{array}{l}\text { Verwachsungen, Darmver- } \\
\text { schluss, chronische abdominel- } \\
\text { le Schmerzen }\end{array}$ & 1,96 & 0,76 & 1,00 & 3,00 & 47,83 & 0,27 & 0,54 \\
\hline Durchschnittswerte & $\mathbf{2 , 4 1}$ & $\mathbf{0 , 6 5}$ & $\mathbf{1 , 1 8}$ & $\mathbf{3 , 0 0}$ & $\mathbf{7 0 , 6 9}$ & $\mathbf{0 , 2 6}$ & $\mathbf{0 , 5 9}$ \\
\hline
\end{tabular}

Tabelle 5: Deskriptive Itemkennwerte zur Bewertungscheckliste „Cholezystektomie“

$M=$ Mittelwert $; S D=$ Standardabweichung; Min = niedrigster erreichter Wert; Max = höchster erreichter Wert; \% = Schwierigkeit in Prozent; $S=$ Trennschärfe; CAD = Cronbachs Alpha, wenn Item weggelassen

\begin{tabular}{|c|c|c|c|c|c|c|c|}
\hline Item & $M$ & SD & Min & Max & $\%$ & $S$ & CAD \\
\hline \multicolumn{8}{|c|}{ Kommunikation } \\
\hline stellt sich mit Namen vor. & 2,98 & 0,15 & 2,00 & 3,00 & 98,85 & & \\
\hline $\begin{array}{l}\text { fragt nach dem aktuellen Befin- } \\
\text { den. }\end{array}$ & 2,29 & 0,87 & 1,00 & 3,00 & 64,62 & 0,20 & 0,53 \\
\hline $\begin{array}{l}\text { tritt den Patientinnen und Pati- } \\
\text { enten wertschätzend gegenüber. }\end{array}$ & 2,94 & 0,24 & 2,00 & 3,00 & 96,95 & 0,35 & 0,48 \\
\hline $\begin{array}{l}\text { hat einen 'roten Faden' in der } \\
\text { Gesprächsführung. }\end{array}$ & 2,69 & 0,48 & 1,00 & 3,00 & 84,52 & 0,33 & 0,45 \\
\hline $\begin{array}{l}\text { verwendet ein angepasstes } \\
\text { Sprechtempo. }\end{array}$ & 2,78 & 0,42 & 2,00 & 3,00 & 88,76 & 0,20 & 0,49 \\
\hline $\begin{array}{l}\text { verwendet verständliche Formu- } \\
\text { lierungen. }\end{array}$ & 2,77 & 0,44 & 1,00 & 3,00 & 88,58 & 0,26 & 0,48 \\
\hline $\begin{array}{l}\text { benutzt den Aufklärungsbogen } \\
\text { zur Veranschaulichung des Ein- } \\
\text { griffs. }\end{array}$ & 2,69 & 0,61 & 1,00 & 3,00 & 84,38 & 0,36 & 0,43 \\
\hline
\end{tabular}




\begin{tabular}{|c|c|c|c|c|c|c|c|}
\hline $\begin{array}{l}\text { hat eine den Patientinnen und } \\
\text { Patienten zugewandte Körper- } \\
\text { haltung. }\end{array}$ & 2,95 & 0,21 & 2,00 & 3,00 & 97,71 & 0,15 & 0,51 \\
\hline hält Blickkontakt. & 2,92 & 0,28 & 2,00 & 3,00 & 95,80 & 0,10 & 0,51 \\
\hline $\begin{array}{l}\text { greift möglicherweise geäußerte } \\
\text { Ängste der Patientinnen und } \\
\text { Patienten auf. }\end{array}$ & 2,72 & 0,52 & 1,00 & 3,00 & 85,83 & 0,24 & 0,48 \\
\hline $\begin{array}{l}\text { gibt mehrfach Gelegenheit für } \\
\text { Nachfragen. }\end{array}$ & 2,70 & 0,55 & 1,00 & 3,00 & 84,77 & 0,15 & 0,51 \\
\hline \multicolumn{8}{|c|}{ Komplikationen } \\
\hline Allergie/Unverträglichkeit & 2,61 & 0,73 & 1,00 & 3,00 & 80,62 & 0,44 & 0,58 \\
\hline $\begin{array}{l}\text { Verletzung von Nachbarorganen } \\
\text { (Darm, Leber, Bauchspeicheldrü- } \\
\text { se) }\end{array}$ & 2,38 & 0,70 & 1,00 & 3,00 & 68,85 & 0,20 & 0,61 \\
\hline Verletzung von Gefäßen & 2,60 & 0,63 & 1,00 & 3,00 & 80,08 & 0,03 & 0,63 \\
\hline $\begin{array}{l}\text { Galleleckage, Gallefisteln, Nar- } \\
\text { benstriktur }\end{array}$ & 1,94 & 0,78 & 1,00 & 3,00 & 47,20 & 0,16 & 0,62 \\
\hline $\begin{array}{l}\text { (Nach-)Blutung, Blutübertragung, } \\
\text { Infektionsrisiko }\end{array}$ & 2,24 & 0,72 & 1,00 & 3,00 & 62,00 & 0,40 & 0,59 \\
\hline $\begin{array}{l}\text { Verletzung von Nerven im Opera- } \\
\text { tionsgebiet }\end{array}$ & 2,62 & 0,70 & 1,00 & 3,00 & 80,77 & 0,23 & 0,61 \\
\hline Lagerungsschäden & 1,26 & 0,64 & 1,00 & 3,00 & 12,90 & $-0,05$ & 0,64 \\
\hline Gewebeschäden durch Strom & 1,21 & 0,57 & 1,00 & 3,00 & 10,40 & 0,09 & 0,62 \\
\hline Übersäuerung/Azidose & 1,95 & 0,96 & 1,00 & 3,00 & 47,27 & 0,17 & 0,62 \\
\hline $\begin{array}{l}\text { Hautemphysem (durch Reste des } \\
\text { Kohlendioxids) }\end{array}$ & 1,85 & 0,95 & 1,00 & 3,00 & 42,58 & 0,33 & 0,59 \\
\hline Schulter-/Bauchschmerzen & 1,37 & 0,70 & 1,00 & 3,00 & 18,50 & 0,38 & 0,59 \\
\hline Pneumothorax, Gasembolie & 1,87 & 0,85 & 1,00 & 3,00 & 43,36 & 0,29 & 0,60 \\
\hline $\begin{array}{l}\text { Wundinfektion, Abszess im Ab- } \\
\text { domen, Peritonitis }\end{array}$ & 2,15 & 0,78 & 1,00 & 3,00 & 57,42 & 0,22 & 0,61 \\
\hline Platzbauch, Narbenbruch & 1,68 & 0,86 & 1,00 & 3,00 & 34,13 & 0,15 & 0,62 \\
\hline Gelbsucht & 1,65 & 0,90 & 1,00 & 3,00 & 32,68 & 0,05 & 0,63 \\
\hline Rest-Steine & 1,40 & 0,75 & 1,00 & 3,00 & 20,16 & 0,30 & 0,60 \\
\hline Thrombose, Embolie & 1,92 & 0,92 & 1,00 & 3,00 & 46,12 & 0,33 & 0,59 \\
\hline $\begin{array}{l}\text { Narbenbildung, kosmetisch un- } \\
\text { günstiges Ergebnis }\end{array}$ & 1,71 & 0,78 & 1,00 & 3,00 & 35,27 & 0,12 & 0,62 \\
\hline $\begin{array}{l}\text { Verwachsungen, Darmverschluss, } \\
\text { chronische abdominelle Schmer- } \\
\text { zen }\end{array}$ & 1,36 & 0,59 & 1,00 & 3,00 & 17,86 & 0,35 & 0,60 \\
\hline mögliche Folgeeingriffe & 2,00 & 0,85 & 1,00 & 3,00 & 50,00 & 0,17 & 0,62 \\
\hline Durchschnittswerte & 2,20 & 0,65 & 1,16 & 3,00 & 59,97 & 0,22 & 0,57 \\
\hline
\end{tabular}


Tabelle 6: Deskriptive Itemkennwerte zur Bewertungscheckliste "Leistenhernie“

$M=$ Mittelwert $; S D=$ Standardabweichung; Min = niedrigster erreichter Wert; Max = höchster erreichter Wert; \% = Schwierigkeit in Prozent; $S=$ Trennschärfe; $C A D=$ Cronbachs Alpha, wenn Item weggelassen

\begin{tabular}{|c|c|c|c|c|c|c|c|}
\hline Item & M & SD & Min & Max & $\%$ & $\mathbf{S}$ & CAD \\
\hline \multicolumn{8}{|c|}{ Kommunikation } \\
\hline stellt sich mit Namen vor. & 2,97 & 0,21 & 1,00 & 3,00 & 98,57 & & \\
\hline fragt nach dem aktuellen Befinden. & 2,40 & 0,83 & 1,00 & 3,00 & 70,14 & 0,21 & 0,70 \\
\hline $\begin{array}{l}\text { tritt den Patientinnen und Patienten wert- } \\
\text { schätzend gegenüber. }\end{array}$ & 2,89 & 0,31 & 2,00 & 3,00 & 94,60 & 0,50 & 0,64 \\
\hline $\begin{array}{l}\text { hat einen 'roten Faden' in der Gesprächsfüh- } \\
\text { rung. }\end{array}$ & 2,61 & 0,56 & 1,00 & 3,00 & 80,37 & 0,50 & 0,62 \\
\hline verwendet ein angepasstes Sprechtempo. & 2,66 & 0,47 & 2,00 & 3,00 & 83,21 & 0,39 & 0,65 \\
\hline verwendet verständliche Formulierungen. & 2,76 & 0,43 & 2,00 & 3,00 & 88,02 & 0,32 & 0,66 \\
\hline $\begin{array}{l}\text { benutzt den Aufklärungsbogen zur Veran- } \\
\text { schaulichung des Eingriffs. }\end{array}$ & 2,51 & 0,70 & 1,00 & 3,00 & 75,72 & 0,13 & 0,71 \\
\hline $\begin{array}{l}\text { hat eine den Patientinnen und Patienten } \\
\text { zugewandte Körperhaltung. }\end{array}$ & 2,89 & 0,33 & 1,00 & 3,00 & 94,57 & 0,55 & 0,63 \\
\hline hält Blickkontakt. & 2,86 & 0,35 & 2,00 & 3,00 & 92,75 & 0,54 & 0,63 \\
\hline $\begin{array}{l}\text { greift möglicherweise geäußerte Ängste des } \\
\text { Patientinnen und Patienten auf. }\end{array}$ & 2,51 & 0,59 & 1,00 & 3,00 & 75,57 & 0,36 & 0,65 \\
\hline gibt mehrfach Gelegenheit für Nachfragen. & 2,64 & 0,59 & 1,00 & 3,00 & 81,75 & 0,39 & 0,64 \\
\hline \multicolumn{8}{|c|}{ Komplikationen } \\
\hline Allergie/Unverträglichkeit & 2,79 & 0,52 & 1,00 & 3,00 & 89,31 & 0,23 & 0,69 \\
\hline Lagerungsschäden & 1,59 & 0,87 & 1,00 & 3,00 & 29,43 & 0,27 & 0,69 \\
\hline Gewebeschäden durch Strom & 1,59 & 0,84 & 1,00 & 3,00 & 29,72 & 0,47 & 0,67 \\
\hline Thrombose, Embolie & 2,65 & 0,69 & 1,00 & 3,00 & 82,29 & 0,07 & 0,71 \\
\hline $\begin{array}{l}\text { Nebenverletzungen (z.B.: Darm, Harnblase, } \\
\text { Samenleiter) }\end{array}$ & 2,37 & 0,76 & 1,00 & 3,00 & 68,66 & 0,33 & 0,68 \\
\hline $\begin{array}{l}\text { Nervenschäden (Taubheitsgefühl, Schmer- } \\
\text { zen, Missempfindungen, motorische Ein- } \\
\text { schränkungen) }\end{array}$ & 2,19 & 0,70 & 1,00 & 3,00 & 59,42 & 0,33 & 0,68 \\
\hline $\begin{array}{l}\text { (Nach-)Blutung, Blutübertragung, Infektions- } \\
\text { risiko }\end{array}$ & 2,37 & 0,67 & 1,00 & 3,00 & 68,62 & 0,15 & 0,70 \\
\hline $\begin{array}{l}\text { Einengung oder Verletzung von Blutgefäßen, } \\
\text { Durchblutungsstörungen des Beines }\end{array}$ & 1,77 & 0,80 & 1,00 & 3,00 & 38,49 & 0,33 & 0,68 \\
\hline Kompartment-Syndrom & 1,24 & 0,58 & 1,00 & 3,00 & 12,13 & 0,26 & 0,69 \\
\hline Hodenschrumpfung/Zeugungsunfähigkeit & 2,02 & 0,91 & 1,00 & 3,00 & 51,05 & 0,33 & 0,68 \\
\hline Sekretverhaltung & 1,39 & 0,69 & 1,00 & 3,00 & 19,71 & 0,46 & 0,67 \\
\hline Wundinfektion & 2,62 & 0,67 & 1,00 & 3,00 & 81,21 & 0,25 & 0,69 \\
\hline Narben, kosmetisch ungünstiges Ergebnis & 2,50 & 0,68 & 1,00 & 3,00 & 75,00 & 0,18 & 0,70 \\
\hline $\begin{array}{l}\text { Nahtbruch an Hohlorganen, Peritonitis, tiefer } \\
\text { Abszess, Fistel }\end{array}$ & 1,50 & 0,70 & 1,00 & 3,00 & 25,00 & 0,22 & 0,69 \\
\hline Platzbauch & 1,71 & 0,91 & 1,00 & 3,00 & 35,71 & 0,49 & 0,66 \\
\hline
\end{tabular}




\begin{tabular}{|c|c|c|c|c|c|c|c|}
\hline Rezidivbruch & 1,96 & 0,82 & 1,00 & 3,00 & 47,84 & 0,12 & 0,70 \\
\hline Darmverschluss & 1,67 & 0,89 & 1,00 & 3,00 & 33,58 & 0,28 & 0,69 \\
\hline $\begin{array}{l}\text { Komplikationen nach Fremdmaterial- } \\
\text { Einpflanzung (Reizergüsse, Unverträglichkeit, } \\
\text { Einbrechen des Netzes in Organe, chron. } \\
\text { Schmerzzustände, Bewegungseinschrän- } \\
\text { kung) }\end{array}$ & 1,57 & 0,70 & 1,00 & 3,00 & 28,52 & 0,20 & 0,69 \\
\hline $\begin{array}{l}\text { Entfernung oder Wechsel des Netz- } \\
\text { Implantats }\end{array}$ & 1,24 & 0,60 & 1,00 & 3,00 & 12,14 & 0,21 & 0,69 \\
\hline Durchschnittswerte & 2,22 & 0,65 & 1,13 & 3,00 & 60,77 & 0,31 & 0,68 \\
\hline
\end{tabular}

\subsection{Deskriptive Auswertung der Ergebnisse}

\subsubsection{Punktescores in den Bewertungschecklisten nach Bewertungsquellen}

Im Rahmen der deskriptiven Auswertung wurden die mittleren Punktescores für alle drei Bewertungschecklisten bzw. deren beiden Skalen in Abhängigkeit der fünf Bewertungsquellen berechnet. In der Skala „Kommunikation“ fiel die Selbsteinschätzung der aufklärenden Person bei allen drei Operationen geringer und damit strenger aus als die Scores der anderen Bewertenden. Hier ergaben sich stets signifikante Unterschiede im Vergleich der aufklärenden Person mit den Peers bzw. der Tutorin/dem Tutor. In der Bewertungscheckliste „Leistenhernie“ waren die Aufklärenden außerdem signifikant strenger als der Experte. In der Bewertungscheckliste "Cholezystektomie“ fiel zudem die Bewertung der Aufklärenden signifikant strenger aus als die der SP. Bemerkenswert ist, dass sich die Bewertungen der studentischen Tutorinnen und Tutoren in keiner der drei Checklisten signifikant vom Experten unterschieden. Um zu beurteilen, ob ein signifikanter Mittelwertunterschied auch eine praktische Relevanz hat, wurde Cohens $d$ berechnet. Die Werte dafür lagen jeweils bei mindestens 0,6 und bedeuteten somit mittlere bis starke Effekte [30]. In der Skala „Komplikationen“ unterschieden sich die Resultate der fünf Bewertungsquellen bei keiner Bewertungscheckliste signifikant voneinander.

Die nachfolgende Abbildung 2 zeigt die Mittelwerte mit Standardabweichungen für die Skalen "Kommunikation" (oben) und "Komplikationen" (unten) der Bewertungschecklisten "Appendektomie" ( $n=186)$, "Cholezystektomie" $(n=121)$ und "Leistenhernie" ( = 135) (von links nach rechts) [31]. 

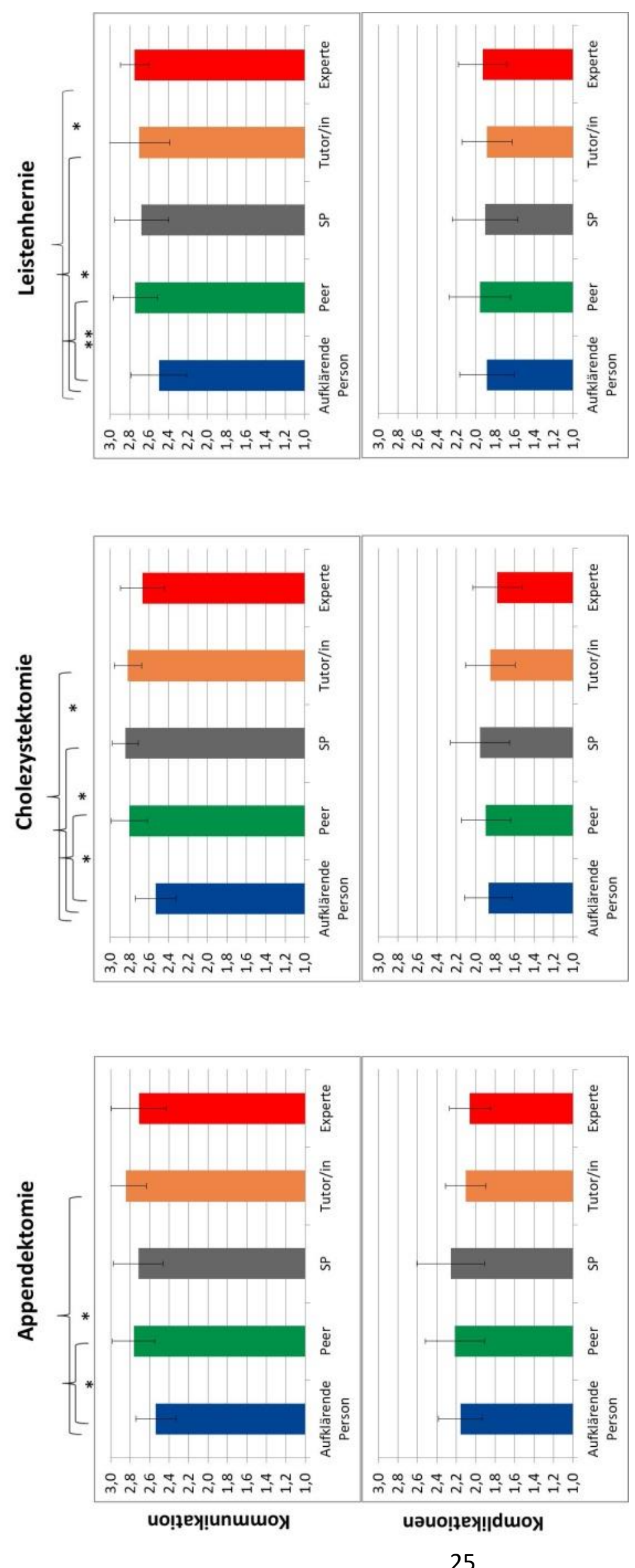


\subsection{2 Übereinstimmung der Bewertungen}

Die Interrater-Reliabilität wurde mittels Intraklassenkorrelationskoeffizienten (ICC) berechnet. Aufgrund der Verwendung identischer Items in der Skala „Kommunikation“ konnten die Ergebnisse über alle drei Bewertungschecklisten zusammengefasst ausgewertet werden. Es zeigte sich eine relative Heterogenität in den Bewertungen, wobei lediglich vier (von insgesamt zehn) Items unterhalb des in der Literatur empfohlenen Schwellenwertes von 0,4 lagen $[32,33]$. In der getrennt ausgewerteten Skala „Komplikationen“ zeigten sich in den Bewertungschecklisten größere Übereinstimmungen mit nur wenigen unterschwelligen Werten. Die Analyse der Übereinstimmung offenbarte, dass über alle Bewertungsquellen hinweg relativ vergleichbare Ergebnisse erzielt wurden. Die nachstehende Abbildung 3 veranschaulicht die Beurteilerübereinstimmung auf Basis der Intraklassenkorrelation (ICC, unjustiert, 95\%Konfidenzintervall) für die Items der Skala „Kommunikation“ (oben, für alle drei Bewertungschecklisten gemeinsam) und der Skala „Komplikationen“ (unten, für die Bewertungschecklisten "Appendektomie", "Cholezystektomie" und "Leistenhernie" getrennt) [31]. 

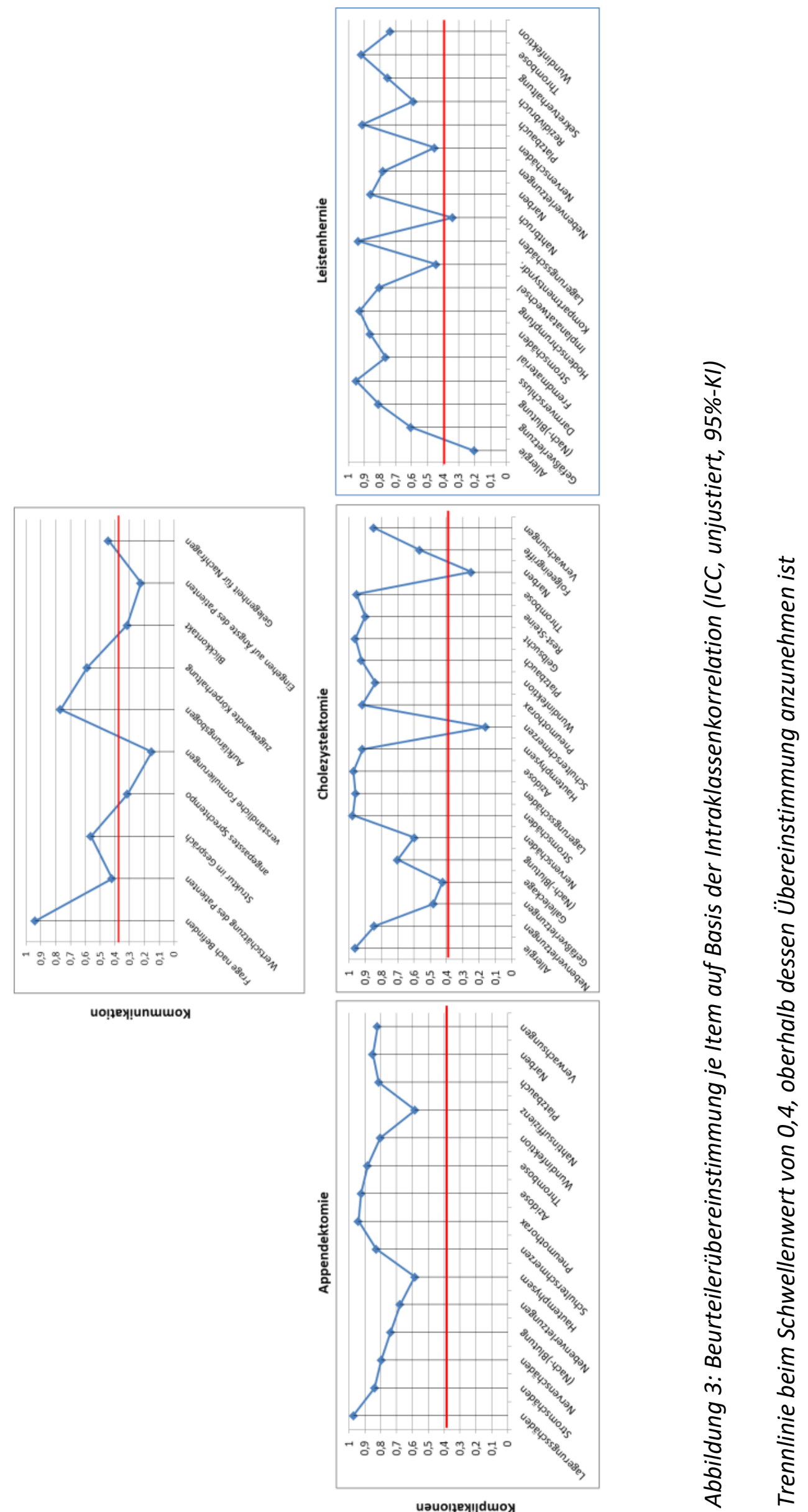


\section{Diskussion}

Die vorliegende Studie konnte die grundsätzliche Durchführbarkeit eines Kommunikationstrainings zur präoperativen Aufklärung mit studentischen Tutorinnen/Tutoren und SP aufzeigen. Die verwendeten Checklisten wiesen hinsichtlich der berechneten Maßzahlen zufriedenstellende Werte auf und konnten deshalb als Grundlage für kompetentes Feedback herangezogen werden. Die Bewertungsunterschiede zwischen den einzelnen Feedbackquellen waren dabei verschwindend gering, vor allem innerhalb der Skala „Komplikationen“. Die hohe Übereinstimmung zwischen den unterschiedlichen Feedbackgebenden wurde durch akzeptable Werte für den Intraklassenkorrelationskoeffizienten untermauert. Gemessen an einem Expertenfeedback stellten sich die Rückmeldungen durch studentische Tutorinnen/Tutoren und SP als sehr zuverlässig heraus.

\subsection{Qualität der Checklisten}

Die Beurteilung bzw. das Feedback, welches den studentischen Aufklärenden im Rahmen des Kommunikationstrainings mitgeteilt wurde, basierte auf einheitlich im Vorfeld angefertigten Checklisten. Insofern war es notwendig zu ermitteln, inwieweit diese Bewertungschecklisten eine adäquate Messgenauigkeit aufwiesen. Diese statistischen Validierungen bezogen sich auf die Berechnung von Cronbachs Alpha sowie Schwierigkeit und Trennschärfe.

\section{Interne Konsistenz}

Die Werte für Cronbachs Alpha für die beiden Subskalen aller drei Bewertungschecklisten liegen zwischen 0,52 und 0,71. In der Literatur findet man unterschiedliche Angaben, ab wann ein Wert als akzeptabel gilt, um von einer intern konsistenten Skala auszugehen. So fordern beispielsweise Bortz und Döring 0,8 als anzustrebenden Wert [34]. Bei Nunnally und Schmitt findet man 0,7 als den üblicherweise angesetzten Wert $[35,36]$. Letzterer weist jedoch auch darauf hin, dass ein niedrigerer Wert nicht zwangsläufig ein Hinderungsgrund für die Verwendung eines Tests sein muss. Entscheidend für die vorliegende Arbeit ist die Tatsache, dass im medizinischen Kontext 
mitunter deutlich mildere Grenzen akzeptiert werden. Dies haben Kiehl et al. in ihrer Arbeit beispielhaft aufgezeigt, welche sich auf die Ausführungen von Möltner et al. stützt $[12,27]$.

Darüber hinaus ist im Rahmen des untersuchten Kommunikationstrainings der inhomogene Wissens- und Erfahrungsstand der teilnehmenden Studierenden zu berücksichtigen. Da die Vorbereitung im Konzept des Flipped Classroom und dies sicher in einem höchst unterschiedlichen Ausmaß erfolgte, ist Cronbachs Alpha in seiner Verwendung als klassischer Homogenitätsparameter kritisch zu betrachten. In Anbetracht der Heterogenität der betrachteten Kohorte verwundert es also nicht, dass in 4 von 6 Subskalen, der "strenge“ Wert von 0,7 für Cronbachs Alpha unterschritten wurde. Selbstverständlich bestünde die Möglichkeit, durch das Entfernen von Items aus der Skala, diese quasi zu homogenisieren, allerdings würde darunter letztlich auch die Qualität der gesamten Skala leiden, wie Yang und Green ausführen [37]. Es blieben dann unter Umständen nämlich nur jene Items zurück, die sich sehr ähnlich sind und dadurch könnte die Qualität der Messung insgesamt leiden. Wir hätten also nach dem Streichen von Items, welche die interne Konsistenz beeinträchtigen, zwar einen höheren Wert für Cronbachs Alpha, die Skala wäre jedoch insgesamt banalisiert [38]. Vorsichtig sollte man laut Yang und Green ohnehin dabei sein, Cronbachs Alpha als Homogenitätsparameter zu verstehen [37]. Schließlich geht es darum, die interne Konsistenz einer Skala zu ermitteln, also aufzuzeigen, inwiefern Items in einer Wechselbeziehung (Schmitt) stehen und dabei handelt es sich nicht um eine bloße Homogenität, was einer Unidimensionalität gleichkommen würde [36, 38].

\section{Schwierigkeit und Trennschärfe}

Bei der Auswertung der Items in Bezug auf Ihre Schwierigkeit stellte sich heraus, dass innerhalb der Subskala „Kommunikation“ die meisten Items von überaus vielen Teilnehmenden erfüllt wurden und deshalb als zu leicht gelten könnten. Dies wiederum führt dazu, dass diese Kommunikationsitems scheinbar geringer differenzieren. Allerdings sollte bei Leistungsüberprüfungen im medizinischen Kontext nicht nur auf eine hohe Reliabilität im Sinne einer zuverlässigen Messung von Unterschieden zwischen 
Studierenden, sondern auch auf die Überprüfung von erbrachten Basisfertigkeiten geachtet werden [27]. Zu diesen „Basisfertigkeiten“ zählten für die Studierenden des 8. Semesters zweifelsohne kommunikative Fertigkeiten. Der Name der Lehrveranstaltung („,Kommunikationstraining“) sowie die Materialien zur Vorbereitung setzten außerdem einen ganz klaren Schwerpunkt. Es wurde im Vorfeld sogar explizit betont, dass es bei den simulierten Aufklärungsgesprächen vorrangig um die Interaktion mit den SP und weniger um das abspulende Aufzählen sämtlicher in den offiziellen Aufklärungsbögen erwähnten Risiken und Komplikationen gehe. Wenn nun also gerade die Items zur Kommunikation bzw. Interaktion von überaus vielen Studierenden erfüllt wurden, darf das durchaus als Positivum der Studie im Sinne einer vorhandenen und erfolgreich nachgewiesenen Kompetenz gewertet werden. In Anbetracht des Würzburger Curriculums hätten wir dies auch nicht anders erwartet. Schließlich haben die Studierenden mit dem Eintritt in das 8. Semester alle wesentlichen Kurse zur ärztlichen Kommunikation, welche sich vom 5. bis zum 7. Semester erstrecken, absolviert. Im Gegensatz dazu konnten wir von den teilnehmenden Studierenden noch nicht erwarten, sämtliche Items der Subskala „Komplikationen“ zu erfüllen. Dies setzt nämlich auch explizit chirurgisches Wissen bzw. Erfahrung bei der Assistenz von Operationen voraus. Da die Vorlesungen und Kurse zum Leistungsnachweis zur Chirurgie jedoch erst im 8. Semester, zum Teil sogar erst am Ende des Semesters stattfanden, überrascht es nicht, dass die Leistung in diesem Bereich geringer ausfiel als bei den Fertigkeiten bezüglich der Kommunikation und Interaktion.

\subsection{Analyse des strukturierten Feedbacks (360-Grad-Feedback)}

Die Technik eines 360-Grad-Feedbacks aus unterschiedlichen Bewertungsquellen wurde ursprünglich für den Bereich großer industrieller Betriebe entwickelt. Während der letzten Jahrzehnte wurde dieses Instrument hingegen auch für den Kontext der medizinischen Aus- und Weiterbildung implementiert [39]. Konkret konnte sogar gezeigt werden, dass ein strukturiertes Multi-Source-Feedback auch in der Auswertung und Verbesserung der Fertigkeiten von chirurgisch tätigen Ärztinnen und Ärzten ein wertvolles Hilfsmittel darstellen kann [40]. In der vorliegenden Studie diente das Feedback aller am Training Beteiligten weniger der Leistungsbeurteilung der Studierenden, als 
vielmehr der Einordnung bzw. Überprüfung des strukturierten Feedbacks selbst. Das heißt, es sollte mit dem Instrument validiert werden, inwieweit die unterschiedlichen Rückmeldungen übereinstimmen. Dies war, wie im Folgenden ausgeführt wird, überwiegend der Fall.

\section{Übereinstimmungen und Diskrepanzen}

Die zentrale Frage unserer Forschungen war, ob es eine ärztliche Expertin/einen ärztlichen Experten als verlässliche Instanz in der Beurteilung vor allem kommunikativer Fähigkeiten Studierender unbedingt braucht. Entsprechend galt unser besonderes Augenmerk den differenziellen Unterschieden zwischen geschulten Tutorinnen bzw. Tutoren einerseits und dem unabhängigen Experten andererseits. Dass sich gerade bezüglich der Skala „Kommunikation“ eine hohe Übereinstimmung zwischen den im Fokus stehenden Bewertenden zeigte, darf im Sinne einer hohen Konstruktvalidität interpretiert werden. Entsprechend darf also das Feedback durch Vertreter der PeerGroup (Tutorinnen und Tutoren) durchaus herangezogen werden, um die gelehrten Inhalte zu festigen bzw. zu evaluieren. Folgt man den Ausführungen von Dochy et al., fördern Instrumente des Peer-Assessments darüber hinaus das Verantwortungsbewusstsein unter den Studierenden [41].

Besonders auffällig bei der Betrachtung der Punktescores in den Bewertungschecklisten ist die durchwegs strenge Selbsteinschätzung der Aufklärenden bezüglich der Skala „Kommunikation“. Folgt man der Literatur, steht dies im Einklang mit der Beobach-

tung, wonach gute Studierende selbstkritisch sind und ihre eigenen Leistungen häufig schlechter einschätzen, als es der Realität entspricht [42]. Dass man im Falle einer Gruppe Medizinstudierender von „guten“ Studierenden sprechen darf, postulieren wir an dieser Stelle großzügig und verweisen dabei unter anderem auf die Zugangshürde des Numerus clausus, welche Bewerberinnen und Bewerber für das Medizinstudium überwinden müssen.

Ein weiterer Einflussfaktor für die Qualität der Selbstbewertung scheint darüber hinaus die eigene tatsächliche Kompetenz zu sein. So fanden Paese und Sniezek heraus, dass 
die Urteilssicherheit über die eigene Leistung mit dem Fachwissen, der Erfahrung und der Größe der Anstrengung für die jeweilige Aufgabe wächst [43]. Bei einer Studie von Mir et al. bewerteten Assistenzärztinnen und -ärzte Videoaufzeichnungen ihrer klinischen Aufgaben kritischer als deren Kolleginnen und Kollegen [44].

Obwohl es nicht im Fokus unserer Untersuchungen stand, so gibt es in der Literatur außerdem Belege für signifikante Geschlechtsunterschiede bezüglich der Selbstbeurteilung von Fähigkeiten. Folgt man Swanson und Lease, schätzten Frauen ihre Kolleginnen in einem Berufseignungstest signifikant höher ein als sich selbst, während die Männer sich selbst tendenziell höher einschätzten als ihre männlichen Kollegen [45]. Es sollte jedoch berücksichtigt werden, dass eine große Zahl von Studien keine geschlechterspezifischen Unterschiede bei der Selbsteinschätzung gefunden hat [46].

Im Alltag lässt sich interessanterweise beobachten, dass sich Menschen bei einfachen und Routinetätigkeiten wie beispielsweise Autofahren oder Tätigkeiten im Haushalt häufig überschätzen. Dies deckt sich auch mit den Erkenntnissen von Moore und Small [47]. Im Kommunikationstraining sahen wir diese überhöhte Selbsteinschätzung vor allem in Bezug auf die Kommunikations-Items und dort speziell bei jenen Punkten, welche auf Empathie, Zugewandtheit oder ganz basale Dinge, wie die Vorstellung mit dem eigenen Namen, abzielen.

Kahneman und Tversky bezeichnen diese Selbstüberschätzung als den sog. Confident Bias. Sie verorten diesen allerdings eher in Bezug auf sehr schwierige und komplexe Situationen [48]. Daneben existiert noch der sog. Dunning-Kruger-Effekt, wonach vor allem Menschen mit geringen Fähigkeiten zu einer Selbstüberschätzung tendierten. Dies habe damit zu tun, dass ein Mangel an Fähigkeiten in einem bestimmten Bereich auch dazu führe, dass es Menschen nicht möglich sei, deren Inkompetenz adäquat einzuschätzen. Die Fähigkeiten, die man nämlich brauche um eine richtige Lösung zu finden, seien genau jene, die auch notwendig seien, um eine Lösung als richtig zu erkennen [49]. 
Vor dem Hintergrund der Beobachtung von Moore und Small müssen wir wohl davon ausgehen, dass die von uns gestellte Aufgabe, ein präoperatives Aufklärungsgespräch zu führen, von den Studierenden als eher komplex empfunden wurde. Trotz der Fülle an betrachteten Vorbehalten wurde für mehr als die Hälfte der Items innerhalb der Skala „Kommunikation“ ein passabler ICC über alle fünf Bewertungsquellen errechnet.

Die Unterschiede zwischen den Bewertungen in Bezug auf die Items der Skala „Komplikationen" fielen ohnehin dermaßen gering aus, dass sich über alle drei Bewertungschecklisten ein zufriedenstellender ICC ergibt. Betrachtet man nur den ICC zur „Appendektomie“, zeigen sich sogar besonders gute Werte von durchwegs über 0,5. Wohlgemerkt wurde stets der strengere, unjustierte ICC berechnet und ein Mindestwert von 0,4 herangezogen [32, 33]. Möglicherweise ist die hohe Übereinstimmung auf die klarere Beurteilungsmöglichkeit der zugrundeliegenden Items zurückzuführen. Während die Nennungen einzelner Komplikationen mitunter einfach zu beobachten und zu dokumentieren ist, lassen sich die Merkmale einer guten Kommunikation begrifflich schwerer fassen.

Insgesamt deckt sich der hohe ICC mit den Ergebnissen von Chenot et al., die 2005 die Qualität der Bewertung durch studentische Tutorinnen und Tutoren im Rahmen einer OSCE untersucht und eine befriedigende Übereinstimmung zwischen geschulten Studierenden und unterrichtenden Ärztinnen und Ärzten festgestellt haben [50]. Die nun vorliegende Studie entwickelte den Vergleich auf Basis eines 360-Grad-Feedbacks konsequent weiter und zeigte abermals auf, dass studentische Tutorinnen und Tutoren eine ebenbürtige Rückmeldung über die Leistungen von Studierenden abgeben können. In der Vergleichsstudie von Kiehl et al. konnte zwar mit einem über alle Bewertungschecklisten hinweg gemessenen ICC von 0,78 eine höhere Übereinstimmung der unterschiedlichen Bewertungsquellen ermittelt werden [12]. Allerdings muss beachtet werden, dass in jener Studie die Leistungen der Studierenden nur durch zwei unterschiedliche Personen bewertet werden, während sich unser 360-Grad-Feedback aus insgesamt fünf unterschiedlichen Bewertungsquellen zusammensetzt. Eine Evaluation zur Qualität des Feedbacks durch studentische Tutorinnen und Tutoren im Kommuni- 
kationsunterricht führten auch Fangauf et al. im Sommersemester 2017 an der Universitätsmedizin Göttingen durch. Im Rahmen einer Hospitation wurde anhand von Checklisten mit 35 Items den Tutorinnen und Tutoren attestiert, dass sie ein sehr gutes Feedback geben, wenngleich die Studierenden selbst den Lernerfolg signifikant höher einschätzten, wenn sie durch Dozentinnen/Dozenten unterrichtet wurden [51]. Der Mehrwert eines Feedbacks durch Studierende in der Lehrendenrolle ergibt sich unter anderem durch das Vorhandensein einer zusätzlichen Ansprechperson auf gleicher Hierarchieebene, die es leichter macht, Fragen zu stellen. Insgesamt dürfte auch die Atmosphäre in einer von Studierenden geleiteten Lehrveranstaltung eine freundschaftlichere sein. Nicht zuletzt können Studierende von Erfahrungen der Kolleginnen und Kollegen mit höherem Wissensstand bzw. aus höheren Semestern mehr profitieren, weil diese unter Umständen auf gewisse Aspekte im Lernprozess eher aufmerksam machen [52].

\section{Videoaufnahme und zweizeitiges Feedbackkonzept}

Als Prüfungsformat zur Evaluation von Fähigkeiten in Hinblick auf eine professionelle ärztliche Kommunikation hat sich in den letzten Jahren vermehrt die OSCE etabliert. Mittlerweile überprüfen die meisten medizinischen Universitäten den Lernerfolg in diesem Bereich mithilfe dieses Formats [53, 54]. Wie zahlreiche Studien ergeben haben, lassen sich kommunikative Fertigkeiten und ärztliche Gesprächsführung hervorragend im Format einer OSCE bewerten. Darüber hinaus wird diese Prüfungsform seitens der Studierenden bereitwillig angenommen, wie ein von Fischbeck et al. über zwei Semester im vorklinischen Abschnitt angelegtes Pilotprojekt im Kursus der Medizinischen Psychologie und Soziologie (Universität Mainz) gezeigt hat. Dort haben die Studierenden angegeben, durch die OSCE in hohem Maße für den Umgang mit den Patientinnen und Patienten dazugelernt zu haben [55]. Auch bei Fischer et al., die Daten im Rahmen eines Kurses „ärztliche Basisfähigkeiten“ (Universität Göttingen) erhoben haben, zeigte sich, dass die Lernziele in den kommunikativen Fähigkeiten von nahezu allen Studierenden erreicht wurden [56]. Tatsächlich sei es sogar seitens des IMPP im Rahmen der Implementierung des nationalen longitudinalen Mustercurriculums Kom- 
munikation in der Medizin explizit gefordert, dass im zweiten Teil der zukünftigen abschließenden klinisch-praktischen Prüfung spezifische kommunikative Kompetenzen im OSCE-Format, durch den Einsatz von Simulationspersonen, geprüft werden [57].

Auch an der Medizinischen Fakultät der Universität Würzburg findet sich seit Jahren ein entsprechender Kurs zur körperlichen Untersuchung im Curriculum. Am Ende dieses Kurses steht eine Prüfung, während der die Studierenden in den einzelnen Modulen durch geschulte Tutorinnen und Tutoren anhand von standardisierten Checklisten beurteilt werden.

Nun weist die vorliegende Studie zum präoperativen Aufklärungsgespräch die Besonderheit auf, dass die aufklärenden Studierenden nicht bloß von geschulten Tutorinnen/Tutoren, sondern unter anderem auch von SP eine Rückmeldung erhalten und sich darüber hinaus selbst in ihrer Rolle als aufklärende Ärztinnen bzw. Ärzte beurteilen sollten. Vor allem Letzteres verlangt nach einer Möglichkeit, sich das eigene Handeln nochmal aus einer „Außenperspektive“ anzusehen. Eine Möglichkeit, wie Studierende das eigene Verhalten besser kritisch reflektieren können, ist der Einsatz von $\mathrm{Vi}$ deoaufnahmen von Gesprächen. Neben der verbesserten Identifikation von Fähigkeiten und Schwächen durch die Studierenden selbst, bieten Videoaufnahmen die Möglichkeit, zeitversetzt auf Reaktionen des Gegenübers (SP) aufmerksam zu werden, welche entweder in der Situation des Gespräches untergegangen sind oder im Rahmen der Rückmeldung durch die SP nicht erwähnt worden sind [58].

Kiehl et al. haben Studierende bei einem Aufklärungstraining sich gegenseitig per Video aufzeichnen lassen. Im Anschluss an diese Gespräche haben sich die Studierenden im Paarverband die Aufnahmen in einem separaten Raum angesehen und auf der Peer-Ebene diskutiert. Ein professionelles Experten-Feedback erfolgte zeitversetzt nach summativer Bewertung anhand von Checklisten [12]. Es gibt jedoch zahlreiche Hinweise, die belegen, dass Studierende am meisten von einer Rückmeldung profitieren, die unmittelbar auf ihr Handeln folgt [59]. Gerade, wenn es darum geht, konkrete Szenarien des klinischen Alltags zu simulieren und das Training in der realen Arbeitsumgebung stattfindet, erscheint ein zeitnahes und konstruktives Feedback insge- 
samt nachhaltiger, weil so die klinische oder simulationsbasierte Lernerfahrung unmittelbar analysiert und diskutiert werden kann. So werden für die erfahrene Situation etwaige Lücken im Wissen und der Durchführung formuliert und neue Lösungsansätze konzipiert, um die Behandlungsqualität nachhaltig zu optimieren. Auf der anderen Seite können positive Verhaltensweisen hervorgehoben werden, was eine zusätzliche Motivation darstellt [60].

In der Zusammenschau kann sich wohl am ehesten ein zweizeitiges und ergänzendes Feedbackkonzept bewähren, wenn im ersten Durchlauf keine Vollständigkeit gewährt werden kann. Deshalb wurden im Rahmen der vorliegenden Studie die Videoaufzeichnungen nicht ausschließlich für die Selbstreflexion der Studierenden verwendet, sondern auch für die zeitversetzte Bewertung durch einen Experten.

\section{Kompetentes Feedback durch Schulung und Checklisten}

Sofern Studierende einen Nutzen aus der Rückmeldung durch SP und studentische Tutorinnen und Tutoren ziehen sollen, kommt der Schulung der Feedbackgebenden eine besondere Bedeutung zu. Es gibt komplexe Untersuchungen, in denen sich widerspiegelt, dass der Sicherstellung der Kompetenz von Tutorinnen und Tutoren in der medizinischen Lehre insofern eine wesentliche Bedeutung zukommt, als dies die Effektivität von Peer Assisted Learning maßgeblich beeinflusst [61]. Im Kontext der vorliegenden Studie bestand die Notwendigkeit einer Schulung der Bewertenden umso mehr, als die studentischen Tutorinnen/Tutoren nicht bloß Lehrinhalte vermittelten, sondern auch Feedback bzw. Bewertungen über Ihre Kommilitoninnen und Kommilitonen abgeben sollten. Nachweise für den Nutzen einer solchen Raterschulung vor einer OSCE wurden im Bereich der Zahnmedizin erbracht, wo unter anderem Haj-Ali et Feil herausgefunden haben, dass durch eine der Prüfung vorausgehende Kalibrierung eine hohe Übereinstimmung mit einem Goldstandard zu erreichen ist [62]. Durch die Etablierung von durch alle Raterinnen und Rater beherrschten Standards, lässt sich außerdem der Prüfungsablauf signifikant optimieren und die Prüfungszeit verkürzen [63]. Inwieweit sich mithilfe einer fundierten Schulung und Vorbereitung die Qualität eines Feedbacks steigern lässt, haben bereits Krautter et al. demonstriert, welche Rückmel- 
dungen zu einer körperlichen Untersuchung sowohl von lehrenden Ärztinnen und Ärzten, als auch von geschulten SP verglichen und beiden eine ähnliche Qualität attestierten [64]. Allerdings wurde in deren Studie die Qualität des Feedbackgebens als solches analysiert, während in der vorliegenden Arbeit lediglich die Inhaltsebene aufgrund der Bewertungen verglichen wurde und nicht die Art und Weise der Übermittlung.

Ein weiteres Problem besteht in der Tatsache, dass sich Kompetenzen der ArztPatienten-Interaktion nicht bloß als richtig oder falsch beurteilen lassen, sondern eines komplexen Evaluationsinstrumentes bedürfen, damit sowohl geschulte Tutorinnen/Tutoren, als auch SP eine standardisierte Rückmeldung geben können. Eine verbale Globalbeurteilung mag zwar einen ausreichenden Einstieg in ein Feedbackgespräch darstellen, allerding erfordert das Setting einer OSCE eine zusätzliche Regulierung auf Basis einer ausgeklügelten Bewertungscheckliste, um die Reliabilität des Feedbacks sicherzustellen [65].

\subsection{Schwächen in der Konzeption}

Mithilfe des E-Learning-Moduls war den Studierenden eine profunde Vorbereitung auf das Kommunikationstraining möglich. Nichtsdestotrotz ist davon auszugehen, dass unter Humanmedizinstudierenden im 8. Semester ein heterogener Wissensstand, insbesondere in Bezug auf chirurgisches Fachwissen, vorliegt. Dies führte verständlicherweise zu differenten Leistungen bei den Aufklärungsgesprächen, speziell bei der Skala „Komplikationen“. Ein weiterer Aspekt, der zu Abweichungen bei den Ergebnissen geführt hat, ist der relative, unterschiedliche Schwierigkeitsgrad der Bewertungschecklisten. So dürfte die Aufklärung zum Eingriff „Appendektomie“ den Studierenden am leichtesten gefallen sein. Deswegen sind dort nicht nur die absoluten Ergebnisse besser, sondern auch die Abweichungen zwischen den Raterinnen/Ratern geringer als beispielsweise bei der "Cholezystektomie“, die insgesamt am schwierigsten erschien.

Daneben wirkte sich auch eine mangelnde Begriffsschulung, insbesondere bei den SP, auf die Güte der Bewertungen aus. So wurde - wie wir auf Nachfrage erfahren haben das Item „Allergie/Unverträglichkeit“ von einzelnen SP im Kontext eines Anamnesegespräches anstatt eines präoperativen Aufklärungsgespräches interpretiert und ent- 
sprechend bewertet. Dies sollte bei zukünftigen Projekten dieser Art Berücksichtigung finden.

Inwieweit sich eine Optimierung durch bessere Schulung der Raterinnen/Rater oder Anpassung der Bewertungschecklisten auswirkt, würde sich durch eine Verlängerung der Studie um ein oder mehrere Semester feststellen lassen. Dass die vorliegende Arbeit sich nur auf ein einzelnes Semester bezieht, darf deshalb auch als Schwäche in der Konzeption interpretiert werden.

Eine Möglichkeit der Evaluation von Checklisten besteht in der Durchführung einer Faktorenanalyse, welche ein Verfahren der Dimensionsreduktion darstellt, indem sie mehrere Items (= Variablen) zu sog. Faktoren zusammenfasst [66]. Eine solche wurde im vorliegenden Fall nicht durchgeführt, da die berechnete Varianz, also die Streuung in den Daten, die von den einzelnen Faktoren (insgesamt zehn) erklärt wird, bei lediglich um die 50 \% für alle drei Bewertungschecklisten lag, sodass dieses Konzept nicht weiter verfolgt wurde [67]. Ungeachtet dieser Tatsache sollte das Verfahren in zukünftigen Studien unbedingt berücksichtigt werden.

Nicht zuletzt muss bemängelt werden, dass die vorliegende Studie lediglich monozentrisch (im Studiengang Humanmedizin der Medizinischen Fakultät der Universität Würzburg) durchgeführt wurde. Um zuverlässige wissenschaftliche Aussagen zur untersuchten Fragestellung treffen zu können, wäre ein weitaus höherer Stichprobenumfang, der nur durch die Durchführung an mehreren Fakultäten oder über einen Zeitraum von mehreren Semestern zu erreichen ist, notwendig [68]. 


\section{Schlussfolgerung}

Das chirurgische Aufklärungsgespräch lässt sich im Rahmen eines im Eigenstudium vorbereiteten und von studentischen Tutorinnen/Tutoren geleiteten Kommunikationstrainings üben. Bei insgesamt überwiegenden Übereinstimmungen im strukturierten Feedback kann der Rückmeldung in erster Linie durch studentische Tutorinnen und Tutoren, aber auch durch Peers der Gruppe zukünftig ein höherer Stellenwert eingeräumt werden. Ferner erscheinen auch SP geeignet, ein adäquates Feedback zu geben. Die Selbstbeurteilung fällt strenger aus, erfüllt aber ebenso den Zweck einer Beurteilung des Leistungsstandes.

Ein Expertenfeedback durch eine anwesende Ärztin/einen anwesenden Arzt mag zwar nach wie vor Wunsch vieler Studierender sein, in Zeiten der Arbeitsverdichtung ist die ärztliche Ressource jedoch limitiert. Daher kann innerhalb eines kalkulierten Settings die Expertin/der Experte durch geschulte Studierende ersetzt werden - vorausgesetzt, der Fokus ist klar umschrieben, was im vorliegenden Kommunikationstraining der Fall war.

Die Tatsache, dass der ärztliche Alltag derart verdichtet ist, dass oftmals der vertraglich festgelegte Umfang an guter Lehre nur erschwert stattfinden kann, soll abschließend ebenfalls Erwähnung finden. Die grundsätzliche Forderung nach ausreichend ärztlichen Ressourcen für die Lehre ist eine äußere Rahmenbedingung, die einen erheblichen Einfluss auf die Unterrichtskonzeption haben kann. 


\section{Zusammenfassung}

Ärztliche Kommunikation wird vielerorts bereits im Studium eingeübt. Das Aufklärungsgespräch vor einer Operation ist ein spezifischer Kommunikationsanlass, der einer differenzierten Rückmeldung an die Studierenden bedarf. Ziel war es, im Rahmen eines Kommunikationstrainings mit SP, die Rückmeldung verschiedener Feedbackgeber (Selbsteinschätzung, Tutorinnen und Tutoren, Peers, SP, ärztlicher Experte) anhand von Bewertungschecklisten zu strukturieren und die Ergebnisse zu vergleichen.

171 Humanmedizinstudierende des 8. Semesters der Universität Würzburg nahmen an einem Training zur präoperativen Aufklärung teil. Im Fokus der Gespräche standen „Kommunikation“ sowie „Risiken/Komplikationen“. Die Vorbereitung erfolgte ausschließlich mittels universitätseigener eLearning-Plattform. Gegenstand der statistischen Auswertungen waren die Testgüte der Bewertungschecklisten, die Scores (Bewertungspunkte) in den Skalen und die Beurteilerübereinstimmung auf Basis des Intraklassenkorrelationskoeffizienten (ICC).

Die Checklisten wiesen zufriedenstellende Werte für interne Konsistenz, Itemschwierigkeit und Trennschärfe auf. Die Mittelwerte der Scores durch die fünf Bewertenden unterschieden sich lediglich hinsichtlich der kommunikativen Fähigkeiten signifikant, wobei hier die Selbsteinschätzung durch die studentischen Aufklärenden am strengsten ausfiel. In Bezug auf die „Risiken und Komplikationen“ gab es eine deutliche Übereinstimmung zwischen Studierenden, Peers, SP, Tutorinnen/Tutoren und Experten.

Es konnte gezeigt werden, dass innerhalb des hochspezifischen Settings eines Simulationstrainings und nach übergelegter Vorbereitung geschulte studentische Tutorinnen/Tutoren eine ähnlich gute Rückmeldung wie der ärztliche Experte geben können. Bei insgesamt überwiegenden Übereinstimmungen im 360-Grad-Feedback darf somit der Rückmeldung durch Tutorinnen/Tutoren oder Peers zukünftig ein höherer Stellenwert eingeräumt werden. 


\section{Literaturverzeichnis}

1. Berendonk C, Beyeler C, Westkämper Rea. Strukturiertes Feedback in der ärztlichen Weiterbildung: Mini-CEX und DOPS. Schweiz Ärzteztg 2008; 89

2. Hattie J, Timperley H. The Power of Feedback. Rev Educ Res 2007; 77: 81-112. doi:10.3102/003465430298487

3. Epstein R, Hundert E. Defining and assesing professional competence. JAMA 2002; 287: 226-235

4. Bundesministerium für Gesundheit. Beschlusstext zum "Masterplan Medizinstudium 2020" Im Internet: https://www.bundesgesundheitsministerium.de/fileadmin/Dateien/4_Pressemitteilun gen/2017/2017_1/170331_Masterplan_Beschlusstext.pdf;

5. Flammang I, Senninger N. Das Aufklärungsgespräch. Allgemein- und Viszeralchirurgie up2date 2018; 12: 135-150. doi:10.1055/a-0586-1253

6. Halbe B. Aufklärungspflicht Teil 1: Rechtssicherheit für Ärzte und Patienten. Dtsch Arztebl International 2017; 114: 858-

7. Ehlers APF, Bitter H. Die ärztliche Behandlung aus juristischer Sicht. Allgemein- und Viszeralchirurgie up2date 2017; 11: 637-657. doi:10.1055/s-0043-122010

8. Teubel A. Arzthaftungsrecht: Aufklären, aber richtig. Dtsch Arztebl International 2010; 107: 951-

9. MFT Medizinischer Fakultätentag der Bundesrepublik Deutschland e. V. Nationaler Kompetenzbasierter Lernzielkatalog Medizin (NKLM) Im Internet:

http://www.nklm.de/files/nklm_final_2015-07-03.pdf;

10. Harden RM, Gleeson FA. Assessment of clinical competence using an objective structured clinical examination (OSCE). Med Educ 1979; 13: 41-54

11. Fischbeck S, Mauch M, Leschnik E et al. Assessment of communication skills with an OSCE among first year medical students. Psychother Psychosom Med Psychol 2011; 61: 465-471. doi:10.1055/s-0031-1291277

12. Kiehl C, Simmenroth-Nayda A, Goerlich $Y$ et al. Standardized and quality-assured video-recorded examination in undergraduate education: informed consent prior to surgery. J Surg Res 2014; 191: 64-73. doi:10.1016/j.jss.2014.01.048

13. Topping KJ. The Effectiveness of Peer Tutoring in Further and Higher Education. A Typology and Review of the Literature. High Educ 1996; 32: 321-345. doi:10.1007/BF00138870

14. Ten Cate O. Practice Report / Bericht aus der Praxis: Peer teaching: From method to philosophy. Z Evid Fortbild Qual Gesundhwes 2017; 127-128: 85-87. doi:10.1016/j.zefq.2017.10.005

15. Bulte C, Betts A, Garner K et al. Student teaching: views of student near-peer teachers and learners. Med Teach 2007; 29: 583-590. doi:10.1080/01421590701583824

16. Werther S. Einführung in Feedbackinstrumente in Organisationen: vom 360-GradFeedback bis hin zur Mitarbeiterbefragung. Wiesbaden: Springer; 2015

17. Knobe $M$, Munker R, Sellei RM et al. Peer teaching: a randomised controlled trial using student-teachers to teach musculoskeletal ultrasound. Med Educ 2010; 44: 148-155. doi:10.1111/j.1365-2923.2009.03557.x

18. Burford B, Illing J, Kergon C et al. User perceptions of multi-source feedback tools for junior doctors. Med Educ 2010; 44: 165-176. doi:10.1111/j.1365-2923.2009.03565.x

19. Lockyer J. Multisource feedback in the assessment of physician competencies. J Contin Educ Health Prof 2003; 23: 4-12. doi:10.1002/chp.1340230103 
20. Wissenschaftsrat. Neustrukturierung des Medizinstudiums und Änderung der Approbationsordnung für Ärzte. Empfehlungen der Expertenkommission zum Masterplan Medizinstudium 2020 (Drs. 7271-18) Im Internet: https://www.wissenschaftsrat.de/download/archiv/7271-18.pdf;

21. Kenner A, Jahn D. Flipped Classroom - Hochschullehre und Tutorien umgedreht gedacht. In Tutorienarbeit im Diskurs III - Qualifizierung für die Zukunft. Münster: WTM Verlag für wissenschaftliche Texte und Medien; 2016: 35-58

22. Feldman $\mathrm{M}$, Lazzara $\mathrm{EH}$, Vanderbilt $\mathrm{AA}$ et al. Rater training to support high-stakes simulation-based assessments. J Contin Educ Health Prof 2012; 32: 279-286. doi:10.1002/chp.21156

23. Simmenroth-Nayda A, Heinemann S, Nolte $C$ et al. Psychometric properties of the Calgary Cambridge guides to assess communication skills of undergraduate medical students. Int J Med Educ 2014; 5: 212-218. doi:10.5116/ijme.5454.c665

24. Wirtz M, Caspar F. Beurteilerübereinstimmung und Beurteilerreliabilität Methoden zur Bestimmung und Verbesserung der Zuverlässigkeit von Einschätzungen mittels Kategoriensystemen und Ratingskalen. Göttingen: Hogrefe; 2002

25. Cortina J. What is Coefficient Alpha? Examination of Theory and Applications. J Appl Psychol 1993; 78: 98-104

26. Schecker H. Überprüfung der Konsistenz von Itemgruppen mit Cronbachs alpha Im Internet:

https://www.springer.com/cda/content/document/cda_downloaddocument/Cronbac h+Alpha.pdf\%3FSGWID=0-0-45-1426184-p175274210; Stand: 22.05 .2018

27. Möltner A, Schellberg D, Jünger J. Grundlegende quantitative Analysen medizinischer Prüfungen. GMS Z Med Ausbild 2006; 23: Doc53

28. [Anonym]. Trennschärfe eines Items. In, Wikipedia

29. Weiss C. Basiswissen Medizinische Statistik [mit Epidemiologie]. 5., überarb. Aufl. Aufl. Heidelberg: Springer; 2010

30. Lenhard W, Lenhard A. Berechnung von Effektstärken. doi:10.13140/RG.2.1.3478.4245

31. Kahr P, Sippel S, Konig S. [Structured Feedback within the Framework of Communication Training in Informed Consent Prior to Surgery]. Zentralbl Chir 2019; 144: 587-596. doi:10.1055/a-1030-4713

32. Fleiss JL. The design and analysis of clinical experiments. New York: Wiley; 1986

33. Fleiss JL. Measuring agreement between two judges on the presence or absence of a trait. Biometrics 1975; 31: 651-659

34. Döring N, Bortz J. Forschungsmethoden und Evaluation in den Sozial- und Humanwissenschaften. 5. vollst. überarb., aktual. und erw. Aufl. Aufl. Berlin: Springer; 2016

35. Nunnally JC. Psychometric theory. 2. Aufl. New York: McGraw-Hill; 1978

36. Schmitt N. Uses and abuses of coefficient alpha. Psychol Assess 1996; 8: 350-353

37. Yang Y, Grenn SB. Coefficient Alpha: A Reliability Coefficient for the 21st Century? J Psychoeduc Assess 2011; 29: 377-392

38. Bauer A. Cronbachs $\alpha$ im Kontext des Grundmodells der klassischen Testtheorie und darüber hinaus; 2015

39. Donnon T, Al Ansari A, Al Alawi S et al. The reliability, validity, and feasibility of multisource feedback physician assessment: a systematic review. Acad Med 2014; 89: 511-516. doi:10.1097/ACM.0000000000000147

40. Violato C, Lockyer J, Fidler H. Multisource feedback: a method of assessing surgical practice. BMJ 2003; 326: 546-548. doi:10.1136/bmj.326.7388.546 
41. Dochy F, Segers M, Sluijsmans D. The use of self-, peer and co-assessment in higher education: A review. Stud High Educ 1999; 24: 331-350.

doi:10.1080/03075079912331379935

42. Boud D, Falchikov N. Quantitative studies of student self-assessment in higher education: a critical analysis of findings. High Educ 1989; 18: 529-549

43. Paese PW, Sniezek JA. Influences on the appropriateness of confidence in judgement: Practice, effort, information and decision making. Organ Behav Hum Decis Process 1991; 48: 100-130

44. Mir MA, Evans RW, Marshall RJ et al. The use of video recordings of medical postgraduates in improving clinical skills. Med Educ 1989; 23: 276-281

45. Swanson JL, H. LS. Gender differences in Self-Ratings of Abilities and Skills. Career Dev Q 1990; 38: 347-359

46. Braun MW. Genauigkeit der Selbsteinschätzung beim Erwerb neuer Kompetenz in Abhängigkeit von Kontrollmeinung, Erfahrung, Selbstaufmerksamkeit, Aengstlichkeit und Geschlecht [Diss Univ Bern, 2003]. Bern; 2003: 1 CD-ROM

47. Moore DA, Small DA. Error and Bias in Comparative Judgment: On Being Both Better and Worse Than We Think We Are. J Pers Soc Psychol 2007; 92: 672-689

48. Kahneman D, Tversky A. Prospect theory: An analysis of decision under risk. Econometrica 1979; 47: 263-291

49. Kruger J, Dunning D. Unskilled and unaware of it: how difficulties in recognizing one's own incompetence lead to inflated self-assessments. J Pers Soc Psychol 1999; 77: 1121-1134. doi:10.1037//0022-3514.77.6.1121

50. Chenot JF, Simmenroth-Nayda A, Koch A et al. Can student tutors act as examiners in an objective structured clinical examination? Med Educ 2007; 41: 1032-1038. doi:10.1111/j.1365-2923.2007.02895.x

51. Fangauf S, Simmenroth A, Herrmann-Lingen C. Studentische Tutoren im Kommunikationsunterricht - Eine Evaluation der Unterrichtsqualität. Deutscher Psychosomatik Kongress; 2018; Berlin

52. Hempel A, Seidl T, Genuchten Ev. Erhebung des Einsatzes von Tutorinnen und Tutoren als Grundlage für zielgerichtete Organisationsentwicklung. Die Hochschullehre 2016; 2

53. Chenot J-F, Ehrhardt M. Objective structured clinical examination (OSCE) in der medizinischen Ausbildung: Eine Alternative zur Klausur. Z Allg Med 2003; 79: 437-442

54. Frey P. Ärztliche Kompetenzen zuverlässig prüfen. OSCE-Prüfungen im Medizinstudium. Beiträge zur Lehrerinnen- und Lehrerbildung 2007; 25: 73-79

55. Fischbeck S, Mauch M, Leschnik E et al. Entwicklung und Evaluation einer OSCE für die Uberprüfung kommunikativer ärztlicher Kompetenz im Kursus der Medizinischen Psychologie und Medizinischen Soziologie. Z Med Psychol 2010; 19: 94-96

56. Fischer T, Chenot J, Kleiber C et al. Kurs "ärztliche Basisfähigkeiten" - Evaluation eines primärärztlich orientierten Unterrichtskonzepts im Rahmen der neuen Approbationsordnung. GMS Z Med Ausbild 2005; 22

57. Hinding B, Gornostayeva M, Lux R et al. Kommunikative Kompetenzen von Ärztinnen und Ärzten. Leitfaden zur Implementierung des nationalen longitudinalen Mustercurriculums Kommunikation in der Medizin In. Mainz: Institut für medizinische und pharmazeutische Prüfungsfragen; 2020

58. Langewitz W. Zur Erlernbarkeit der Arzt-Patienten-Kommunikation in der Medizinischen Ausbildung. Bundesgesundheitsblatt 2012; 55: 1176-1182. doi:10.1007/s00103-012-1533-0

59. Norcini J, Burch V. Workplace-based assessment as an educational tool: AMEE Guide No. 31. Med Teach 2007; 29: 855-871. doi:10.1080/01421590701775453 
60. St. Pierre M, Breuer G. Simulation in der Medizin Grundlegende Konzepte - Klinische Anwendung. 2. Auflage. Aufl

61. Dolmans DH, Wolfhagen IH. Complex interactions between tutor performance, tutorial group productivity and the effectiveness of PBL units as perceived by students. Adv Health Sci Educ Theory Pract 2005; 10: 253-261. doi:10.1007/s10459-005-0665-5

62. Haj-Ali R, Feil P. Rater reliability: short- and long-term effects of calibration training. J Dent Educ 2006; 70: 428-433

63. McAndrew R, Chadwick B, Treasure ET. The influence of a short training program on the clinical examination of dental restorations. Oper Dent 2011; 36: 143-152. doi:10.2341/10-202-C

64. Krautter M, Diefenbacher K, Schultz JH et al. Physical examination skills training: Faculty staff vs. patient instructor feedback-A controlled trial. PLoS One 2017; 12: e0180308. doi:10.1371/journal.pone.0180308

65. Newble D. Techniques for measuring clinical competence: objective structured clinical examinations. Med Educ 2004; 38: 199-203. doi:10.1111/j.1365-2923.2004.01755.x

66. Moosbrugger $\mathrm{H}$, Schermelleh-Engel K. Exploratorische (EFA) und Konfirmatorische Faktorenanalyse (CFA). In: Moosbrugger H, Kelava A, Hrsg. Testtheorie und Fragebogenkonstruktion. Berlin, Heidelberg: Springer; 2012. doi:https://doi.org/10.1007/978-3-642-20072-4_13

67. Support für Minitab 18. Interpretieren aller Statistiken und Grafiken für Faktorenanalyse Im Internet: https://support.minitab.com/de-de/minitab/18/helpand-how-to/modeling-statistics/multivariate/how-to/factor-analysis/interpret-theresults/all-statistics-and-graphs/;

68. Santis M, Steininger T. Monozentrische Studie. In: Lenk C, Duttge G, Fangerau H, Hrsg. Handbuch Ethik und Recht der Forschung am Menschen. Berlin, Heidelberg: Springer; 2014. doi:https://doi.org/10.1007/978-3-642-35099-3_98 


\section{Anhang A: Rollen der SP}

\section{Appendizitis - Frau/Herr Körte}

\section{OP-Aufklärung:}

Laparoskopische Appendektomie (Entfernung des Wurmfortsatzes mittels ,Schlüssellochtechnik')

\section{Krankheitsbezogene Anamnese:}

Sie (Ihr reales Alter) kamen mittags in die Notfallaufnahme, nachdem Sie vor etwa 5 Stunden am Morgen von Unterbauchschmerzen aufgeweckt worden waren. Sie sind nicht zur Uni/zur Arbeit/zum Einkaufen gegangen und haben sich zunächst wieder ins Bett gelegt. Sie verspürten Übelkeit und mussten zweimal erbrechen. Stuhlgang und Wasserlassen waren bis zum Schmerzereignis unauffällig. Sie haben am Morgen um ca. 8 Uhr 2 Tabletten Ibuprofen eingenommen, in der Hoffnung, doch noch aus dem Haus gehen zu können. Sie leiden an Migräne, nehmen bei Bedarf Dolortriptan ein und sind noch nie operiert worden.

Sie haben Schmerzen beim Gehen, halten sich den rechten Unterbauch mit Schonhaltung vornübergebeugt, wenn Sie sich bewegen müssen. Der Oberarzt hat eine akute Appendizitis festgestellt und Ihnen bereits mitgeteilt, dass die Operation in den nächsten 2-3 Stunden stattfinden wird. Das Sitzen ist unangenehm und Sie werden während des Aufklärungsgespräches bei kleineren Bewegungen immer wieder unangenehm an den Schmerz erinnert.

Sozialanamnese: Bitte Ausbildungsstand/Beruf und Hobbies improvisieren

\section{Familienanamnese/Eigenanamnese:}

Sie sind noch nie operiert worden. Sie nehmen nur eine Bedarfsmedikation ein (s.o.). Ihre Mutter hatte Brustkrebs. Ihr älterer Bruder hatte einen langen Krankenhausaufenthalt nach ,Blinddarmdurchbruch'. 
Risikoanamnese: Kein Nikotin, gelegentlich Alkohol.

Aufgabe:

Hören Sie aufmerksam zu, wenn der Arzt/die Ärztin Ihnen die Operation erklärt. Unterbrechen Sie nur dann, wenn Sie etwas nicht verstehen, z.B. wenn lateinische Ausdrücke verwendet werden. Fragen Sie am Ende nach, wenn Sie wichtige Dinge nicht verstanden haben.

Auf jeden Fall sollte am Schluss beantwortet sein (falls nicht, bitte nachfragen):

- Mit welchem Gas wird mein Bauch aufgepumpt?

- Muss die Operation mit der Schlüssellochtechnik durchgeführt werden?

- Kann bei der Operation die Harnblase verletzt werden?

- Kann ich eine Woche nach OP meiner normalen Tätigkeit bzw. bestimmten Verpflichtungen (bitte improvisieren) nachgehen?

Bitte keine übertriebene Angst spielen, sondern nur „leicht ängstlich“.

\section{Gallensteinleiden - Frau/Herr Weber}

\section{OP-Aufklärung:}

Laparoskopische Cholezystektomie (Entfernung der Cholezystektomie mittels ,Schlüssellochtechnik')

\section{Krankheitsbezogene Anamnese:}

Sie (Ihr reales Alter) haben schon seit mehr als einem Jahr gelegentlich ein Druckgefühl im rechten Oberbauch. Vor ca. 6 Wochen sind Sie nach einer Geburtstagsfeier mit gürtelförmigen, teils krampfartigen Oberbauchschmerzen in die Notfallaufnahme des Klinikums gegangen, weil Sie es zu Hause trotz mehrerer Aspirin-Tabletten nicht mehr aushalten konnten. Sie wurden stationär aufgenommen und haben von einem ,Internisten' eine ERCP (endoskopische Untersuchung der Gallenwege über eine Magenspiegelung) erhalten. Letztlich wurden bei Ihnen 2 kleine Steine aus dem Gallengang entfernt. 
Seither haben Sie Ihre Ernährung umgestellt, essen jetzt eher fettarm und es ging Ihnen gut dabei. Nun werden Sie vom Hausarzt geschickt, da noch Steine in der Cholezystektomie sind, wie der Hausarzt per Ultraschall festgestellt hat. Aktuell haben Sie keine Bauchschmerzen.

Sozialanamnese: Beruf und Hobbies bitte improvisieren.

\section{Familienanamnese/Eigenanamnese:}

Ihre Mutter hatte auch Gallensteine. Sie sind vor 2 Jahren nach einem Fahrradsturz an einem Unterarmbruch rechts operiert worden. Sie nehmen keine regelmäßigen Medikamente ein.

Risikoanamnese: Kein Nikotin, gelegentlich Alkohol zu Feierlichkeiten.

\section{Aufgabe:}

Hören Sie aufmerksam zu, wenn der Arzt/die Ärztin Ihnen die Operation erklärt. Unterbrechen Sie nur dann, wenn Sie etwas nicht verstehen, z.B. wenn lateinische Ausdrücke verwendet werden. Fragen Sie am Ende nach, wenn Sie wichtige Dinge nicht verstanden haben.

Auf jeden Fall sollte am Schluss beantwortet sein (falls nicht, bitte nachfragen):

- Was passiert, wenn der Hauptgallengang bei der OP verletzt wird?

- Ich bin in 10 Tagen auf einer Hochzeit eingeladen, bin ich bis dahin wieder fit und kann tanzen?

- Kann ich nach der Operation wieder gegrilltes Fleisch essen?

- Können die verbliebenen Steine nicht mit „Stoßwellen zertrümmert“ werden? Bei einem Freund ist das auch so mit Nierensteinen gemacht worden.

Bitte keine übertriebene Angst spielen, sondern nur „leicht ängstlich“. 


\section{Leistenbruch - Frau/Herr Bäcker}

OP-Aufklärung:

Traditioneller (offener) Hernienverschluss mit Kunststoffnetzeinlage (OP nach Lichtenstein)

Anamnese: Sie (Ihr reales Alter) stellten sich zunächst am vergangenen Wochenende mit einer schmerzhaften Schwellung in der linken Leiste in der Notfallaufnahme vor. Sie hatten Ihrer Tochter beim Umzug geholfen. Außerdem ist Ihnen aufgefallen, dass die linke Leiste schon seit einiger Zeit nach dem Treppensteigen und anderen körperlichen Belastungen (insb. Ihres geliebten Sports) etwas geschmerzt hat. Die Schwellung ist nur gelegentlich da und lässt sich dann ohne größere Mühe wegdrücken, das Manöver ist aber unangenehm und es ,flutscht' kurzfristig. Sie kommen jetzt in die chirurgische Poliklinik zur Vereinbarung eines OP-Termins, so war es in der Notaufnahme auch mit dem damals diensthabenden Facharzt besprochen worden. Sie wünschen einen traditionellen Hernienverschluss (offenes Verfahren) zur Einlage des stabilisierenden Kunststoffnetzes. Ihr Schwager hat mit dem minimal-invasiven Verfahren ,Pech gehabt'. Es ist bei ihm zu einem frühen Wiederauftreten des Bruches gekommen und er musste ein zweites Mal operiert werden.

\section{Sozialanamnese:}

Beruf und Hobbies (u.a. Fahrradfahren) improvisieren.

\section{Eigenanamnese/Familienanamnese:}

Sie leiden an Asthma, fühlen sich bei körperlicher Belastung und dann Kurzatmigkeit deutlich eingeschränkt und müssen regelmäßig Bronchosprays applizieren. Sie sind als Kind einmal am Blinddarm operiert worden, sonst noch nicht. Deshalb sind Sie ängstlich, weil Operationen für Sie etwas ganz Unbekanntes sind. In der Familie gibt es keine chronischen Erkrankungen. Aber Sie haben diverse Allergien gegen Gräser, Nüsse, braunes Pflaster und Nickel. 


\section{Risikoanamnese:}

Kein Nikotin, gelegentlich Alkohol.

Aufgabe:

Hören Sie aufmerksam zu, wenn der Arzt/die Ärztin Ihnen die Operation erklärt. Unterbrechen Sie nur dann, wenn Sie etwas nicht verstehen, z.B. wenn lateinische Ausdrücke verwendet werden. Fragen Sie am Ende nach, wenn Sie wichtige Dinge nicht verstanden haben.

Auf jeden Fall sollte am Schluss beantwortet sein (falls nicht, bitte nachfragen):

- Ich habe viele Allergien, werde ich denn das Netz vertragen?

- Ich habe gehört, dass es auch Operationstechniken gibt, bei denen KEIN Kunststoffnetz eingelegt wird. Warum empfehlen Sie mir das nicht?

- Wann kann ich nach der OP wieder Fahrradfahren?

- Ich bin allergisch gegen braunes Pflaster, was werden Sie verwenden?

Bitte keine übertriebene Angst spielen, sondern nur „leicht ängstlich“. 


\section{Anhang B: Bewertungschecklisten}

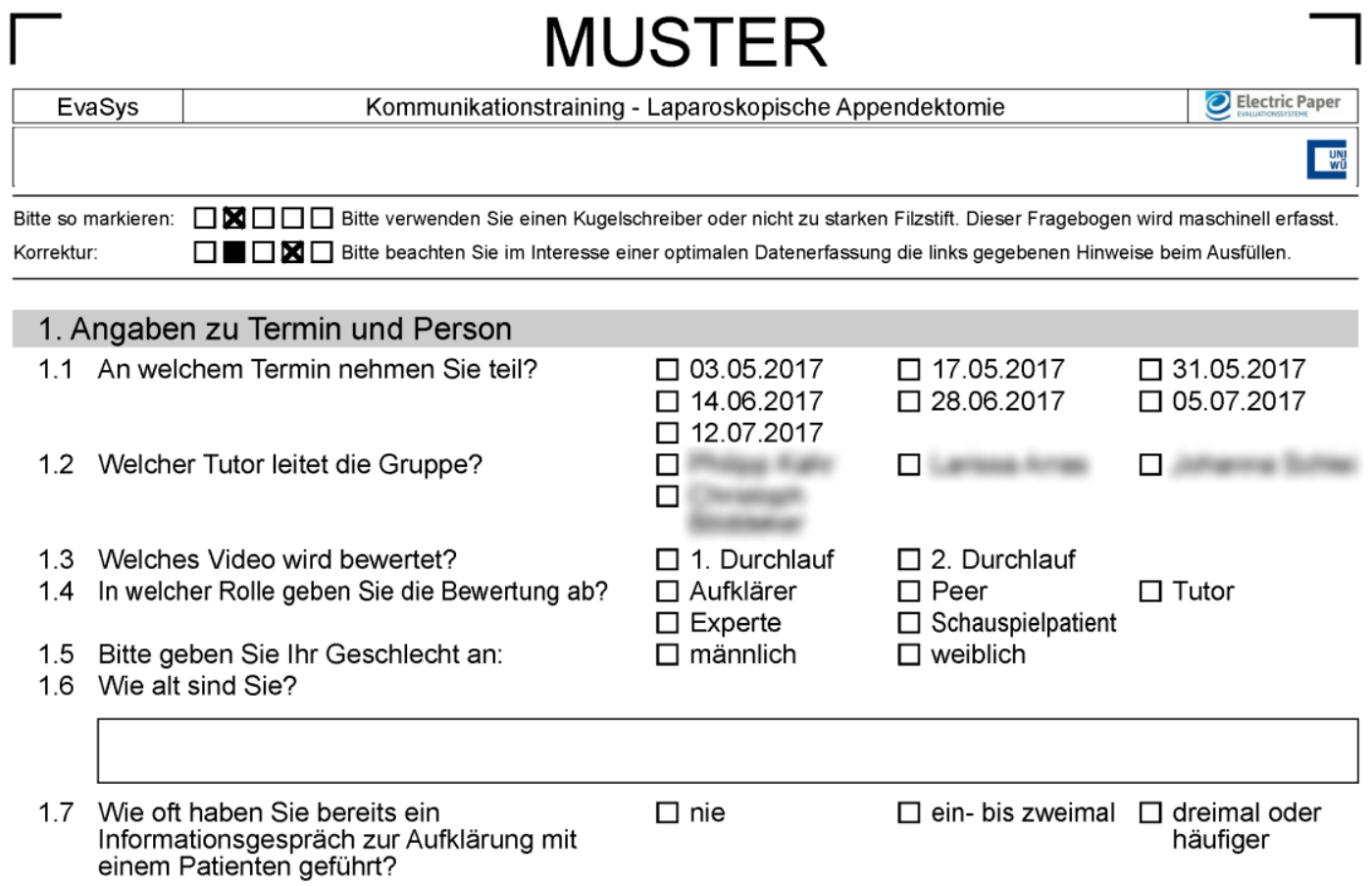

\section{Kommunikative Kompetenz und Interaktion mit Schauspielpatient}

2.1 stellt sich mit Namen vor.

2.2 ... fragt nach dem aktuellen Befinden.

2.3 ... tritt dem Patienten wertschätzend gegenüber.

2.4 ... hat einen "roten Faden" in der Gesprächsführung.

2.5 ... verwendet ein angepasstes Sprechtempo.

2.6 ... verwendet verständliche Formulierungen.

$2.7 \ldots$ benutzt den Aufklärungsbogen zur

Veranschaulichung des Eingriffs.

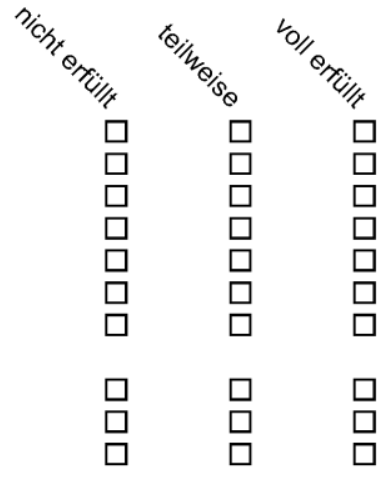

2.9 ... hält Blickkontakt.

$2.10 \ldots$ greift möglicherweise geäußerte Ängste des

Patienten auf.

2.11 ... gibt mehrfach Gelegenheit für Nachfragen.

\section{Einstieg in das Aufklärungsgespräch}

3.1 ... erklärt, was eine Blinddarmentzündung ist.

3.2 ... erläutert, weshalb operiert werden muss.

3.3 ... stellt die Dringlichkeit der OP heraus.

$3.4 \ldots$ beschreibt die Operationsmethode.

3.5 ... skizziert Vor- und Nachteile des laparoskopischen Vorgehens.

3.6 ... erwähnt die Möglichkeit des Umsteigens auf die offene Methode. 


\section{MUSTER}

\begin{tabular}{|l|l|l|l|l} 
EvaSys & Kommunikationstraining - Laparoskopische Appendektomie & Electric Paper \\
\hline
\end{tabular}

\section{Einstieg in das Aufklärungsgespräch [Fortsetzung]}

3.7 ... spricht die Suche nach anderen Krankheitsherden (z.B. Meckel-Divertikel) an.

\section{Komplikationen}

* "voll erfüllt" nur, wenn alle Komplikationen genannt werden

4.1 Allergie/Unverträglichkeit

4.2 Lagerungsschäden

4.3 Gewebeschäden durch Strom

4.4 Verletzung von Nerven im Operationsgebiet

4.5 (Nach-)Blutung, Blutübertragung, Infektionsrisiko*

4.6 Verletzung von Nachbarorganen (Darm, Harnblase, innere Genitale)*

4.7 Verletzung von Gefäßen, insb. beim Setzen der Trokare

4.8 Hautemphysem (durch Reste des Kohlendioxids)

4.9 Schulter-/Bauchschmerzen

4.10 Pneumothorax, Gasembolie*

4.11 Übersäuerung/Azidose

4.12 Thrombose, Embolie*

4.13 Wundinfektion, Abszess im Abdomen, Peritonitis*

4.14 Nahtinsuffizienz, Stuhlfisteln*

4.15 Platzbauch, Narbenbruch*

4.16 Narbenbildung, kosmetisch ungünstiges Ergebnis*

4.17 Verwachsungen, Darmverschluss, chronische abdominelle Schmerzen*

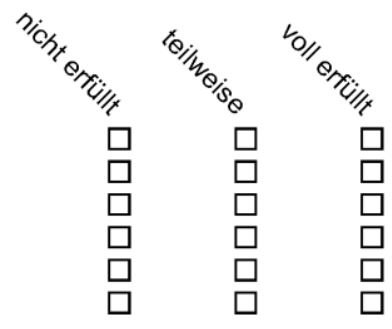

\section{Postoperative Verhaltensmaßnahmen}

5.1 ... kündigt den zügigen Kostaufbau an.

$5.2 \ldots$ spricht über körperliche/sportliche Aktivitäten nach dem Eingriff.

\section{Globalbewertung}

6.1 Das Aufklärungsgespräch bewerte ich insgesamt mit sehr gut $\quad \begin{aligned} & \square \\ & \quad\end{aligned} \quad \square \quad \square$ mangelhaft der Note ...

6.2 Was fand ich besonders gut am Kommunikationstraining?

6.3 Was fand ich weniger gut und könnte verbessert werden? 
$\bar{T}$

MUSTER

\begin{tabular}{l|c|c|}
\hline \multicolumn{1}{|c|}{ EvaSys } & Kommunikationstraining - Laparoskopische Cholezystektomie & Electric Paper \\
\hline \hline & & \\
\hline Bitte so markieren: & $\square \square \square \square$ Bitte verwenden Sie einen Kugelschreiber oder nicht zu starken Filzstift. Dieser Fragebogen wird maschinell erfasst. \\
Korrektur: & $\square \square \square \square$ Bitte beachten Sie im Interesse einer optimalen Datenerfassung die links gegebenen Hinweise beim Ausfüllen.
\end{tabular}

\section{Angaben zu Termin und Person}

1.1 An welchem Termin nehmen Sie teil?

$\square 03.05 .2017$

$\square$ 12.07.2017

1.2 Welcher Tutor leitet die Gruppe?

1.3 Welches Video wird bewertet?

1.4 In welcher Rolle geben Sie die Bewertung ab?

1.5 Bitte geben Sie Ihr Geschlecht an

1.6 Wie alt sind Sie? $\square$

$\square$ 1. Durchlauf

$\square$ Aufkläre

$\square$ Experte

$\square$ männlich $\square$ 17.05.2017

$\square$ 28.06.2017

$\square$

$\square$ 2. Durchlauf

$\square$ Peer

$\square$ Schauspielpatient

$\square$ weiblich $\square$ 31.05.2017

$\square 05.07 .2017$

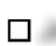

$\square$ Tutor

1.7 Wie oft haben Sie bereits ein Informationsgespräch zur Aufklärung mit

$\square$ nie

$\square$ ein- bis zweimal $\square$ dreimal oder häufiger

\section{Kommunikative Kompetenz und Interaktion mit Schauspielpatient}

2.1 ... stellt sich mit Namen vor.

2.2 ... fragt nach dem aktuellen Befinden.

2.3 ... tritt dem Patienten wertschätzend gegenüber

2.4 ... hat einen "roten Faden" in der Gesprächsführung.

2.5 ... verwendet ein angepasstes Sprechtempo.

2.6 ... verwendet verständliche Formulierungen.

2.7 ... benutzt den Aufklärungsbogen zur

Veranschaulichung des Eingriffs.

2.8 ... hat eine dem Patienten zugewandte Körperhaltung

2.9 ... hält Blickkontakt.

2.10 ... greift möglicherweise geäußerte Ängste des

Patienten auf.

2.11 ... gibt mehrfach Gelegenheit für Nachfragen.

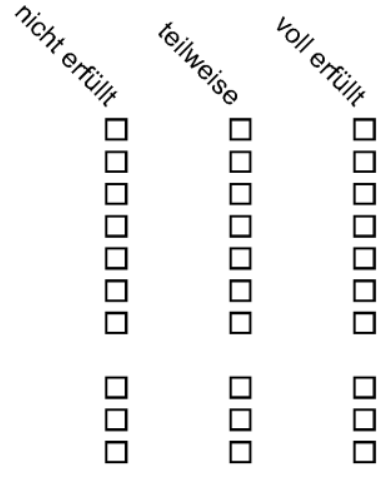

3. Einstieg in das Aufklärungsgespräch

3.1 ... erläutert die Diagnose.

3.2 ... erklärt, weshalb operiert werden muss.

3.3 ... betont, dass es sich um einen elektiven Eingriff handelt.

3.4 ... beschreibt die Operationsmethode.

3.5 ... skizziert Vor- und Nachteile des laparoskopischen Vorgehens.

3.6 ... erwähnt die Möglichkeit des Umsteigens auf die offene Methode. 


\section{Einstieg in das Aufklärungsgespräch [Fortsetzung]}

3.7 ... spricht die etwaige Operation an den Gallenwegen oder die Entfernung von Lebergewebe an.

\section{Komplikationen}

* "voll erfüllt" nur, wenn alle Komplikationen genannt werden

4.1 Allergie/Unverträglichkeit

4.2 Verletzung von Nachbarorganen (Darm, Leber, Bauchspeicheldrüse)*

4.3 Verletzung von Gefäßen

4.4 Galleleckage, Gallefisteln, Narbenstriktur*

4.5 (Nach-)Blutung, Blutübertragung, Infektionsrisiko*

4.6 Verletzung von Nerven im Operationsgebiet

4.7 Lagerungsschäden

4.8 Gewebeschäden durch Strom

4.9 Übersäuerung/Azidose

4.10 Hautemphysem (durch Reste des Kohlendioxids)

4.11 Schulter-/Bauchschmerzen

4.12 Pneumothorax, Gasembolie*

4.13 Wundinfektion, Abszess im Abdomen, Peritonitis*

4.14 Platzbauch, Narbenbruch*

4.15 Gelbsucht

4.16 Rest-Steine

4.17 Thrombose, Embolie*

4.18 Narbenbildung, kosmetisch ungünstiges Ergebnis*

4.19 Verwachsungen, Darmverschluss, chronische abdominelle Schmerzen*

4.20 mögliche Folgeeingriffe

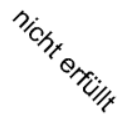

$\square \quad \square \quad \square$

$\square$

\section{Postoperative Verhaltensmaßnahmen}

\section{1 ... kündigt den zügigen Kostaufbau an.}

5.2 ... zeigt die langfristige Vermeidung fettreicher, opulenter Speisen auf.

\section{Globalbewertung}

6.1 Das Aufklärungsgespräch bewerte ich insgesamt mit sehr gut $\quad \square \quad \square \quad \square \quad \square \quad \square$ mangelhaft der Note ...

6.2 Was fand ich besonders gut am Kommunikationstraining?

6.3 Was fand ich weniger gut und könnte verbessert werden? 


\section{MUSTER}

\begin{tabular}{l|c|c|}
\hline \multicolumn{1}{|c|}{ EvaSys } & Kommunikationstraining - Leistenhernienverschluss & Electric Paper \\
\hline \hline & & \\
\hline Bitte so markieren: & $\square \square \square \square$ Bitte verwenden Sie einen Kugelschreiber oder nicht zu starken Filzstift. Dieser Fragebogen wird maschinell erfasst. \\
Korrektur: & $\square \square \square \square$ Bitte beachten Sie im Interesse einer optimalen Datenerfassung die links gegebenen Hinweise beim Ausfüllen. \\
\hline
\end{tabular}

\section{Angaben zu Termin und Person}

1.1 An welchem Termin nehmen Sie teil?

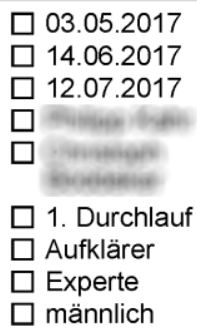

$\square$ 17.05.2017

$\square$ 31.05.2017

$$
\square \text { 14.06.2017 }
$$

$\square$ 28.06.2017

$\square 05.07 .2017$

1.2 Welcher Tutor leitet die Gruppe?

1.3 Welches Video wird bewertet?

1.4 In welcher Rolle geben Sie die Bewertung ab?

\section{urchlauf \\ $\square$ Experte \\ $\square$ männlich}

.5 Bitte geben Sie Ihr Geschlecht an:
$\square$ 2. Durchlauf
$\square$ Peer
$\square$ Schauspielpatient
$\square$ weiblich

1.6 Wie alt sind Sie?

1.7 Wie oft haben Sie bereits ein Informationsgespräch zur Aufklärung mit

$\square$ nie einem Patienten geführt?

\section{Kommunikative Kompetenz und Interaktion mit Schauspielpatient}

2.1 ... stellt sich mit Namen vor.

2.2 ... fragt nach dem aktuellen Befinden.

2.3 ... tritt dem Patienten wertschätzend gegenüber.

2.4 ... hat einen "roten Faden" in der Gesprächsführung.

2.5 ... verwendet ein angepasstes Sprechtempo.

2.6 ... verwendet verständliche Formulierungen.

$2.7 \ldots$ benutzt den Aufklärungsbogen zur

Veranschaulichung des Eingriffs.

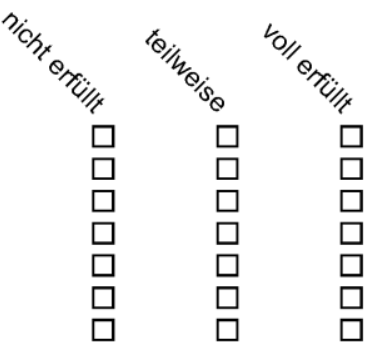

2.8 ... hat eine dem Patienten zugewandte Körperhaltung

2.9 ... hält Blickkontakt.

2.10 ... greift möglicherweise geäußerte Ängste des

Patienten auf.

2.11 ... gibt mehrfach Gelegenheit für Nachfragen.

\section{Einstieg in das Aufklärungsgespräch}

3.1 ... erläutert, was ein Leistenbruch ist.

3.2 ... erklärt, weshalb operiert werden muss.

3.3 ... betont, dass es sich um einen elektiven Eingriff handelt.

3.4 ... beschreibt die Operationsmethode.

3.5 ... skizziert Vor- und Nachteile gegenüber der minimal-invasiven Methode.

3.6 ... erwähnt die Möglichkeit der Eröffnung der Bauchhöhle bei Brucheinklemmung.

3.7 ... spricht über die Möglichkeit einer Darmresektion. 


\section{MUSTER}

EvaSys $\quad$ Kommunikationstraining - Leistenhernienverschluss

\section{Komplikationen}

* "voll erfüllt" nur, wenn alle Komplikationen genannt werden

4.1 Allergie/Unverträglichkeit

4.2 Lagerungsschäden

4.3 Gewebeschäden durch Strom

4.4 Thrombose, Embolie*

4.5 Nebenverletzungen (z.B.: Darm, Harnblase, Samenleiter)

4.6 Nervenschäden (Taubheitsgefühl, Schmerzen,

Missempfindungen, motorische Einschränkungen)*

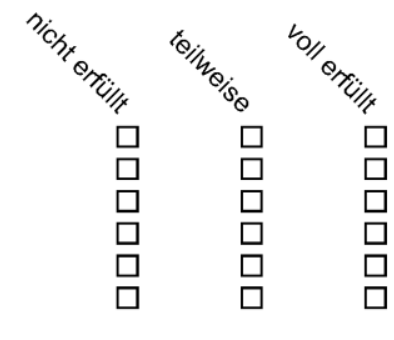

4.7 (Nach-)Blutung, Blutübertragung, Infektionsrisiko*

4.8 Einengung oder Verletzung von Blutgefäßen,

$\square \quad \square \quad \square$

Durchblutungsstörungen des Beines ${ }^{\star}$

4.9 Kompartment-Syndrom

4.10 Hodenschrumpfung/Zeugungsunfähigkeit

4.11 Sekretverhaltung

4.12 Wundinfektion

4.13 Narben, kosmetisch ungünstiges Ergebnis*

4.14 Nahtbruch an Hohlorganen, Peritonitis, tiefer Abszess, Fistel ${ }^{*}$

4.15 Platzbauch

4.16 Rezidivbruch

4.17 Darmverschluss

4.18 Komplikationen nach Fremdmaterial-Einpflanzung

(Reizergüsse, Unverträglichkeit, Einbrechen des

Netzes in Organe, chron. Schmerzzustände,

Bewegungseinschränkung)*

4.19 Entfernung oder Wechsel des Netz-Implantats

$\begin{array}{lll}\square & \square & \square \\ \square & \square & \square \\ \square & \square & \square \\ \square & \square & \square \\ \square & \square & \square \\ \square & \square & \square\end{array}$

\section{Postoperative Verhaltensmaßnahmen}

5.1 ... kündigt den zügigen Kostaufbau an.

5.2 ... spricht über sportliche Aktivitäten nach der OP.

$\begin{array}{lll}\square & \square & \square \\ \square & \square & \square \\ \square & \square & \square \\ \square & \square & \square\end{array}$

\section{Globalbewertung}

6.1 Das Aufklärungsgespräch bewerte ich insgesamt mit sehr gut $\quad \begin{aligned} & \square \quad \\ & \quad\end{aligned} \quad \square \quad$ mangelhaft der Note...

6.2 Was fand ich besonders gut am Kommunikationstraining?

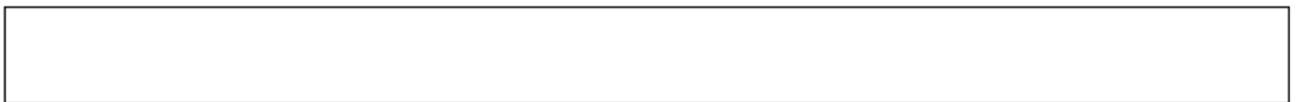

6.3 Was fand ich weniger gut und könnte verbessert werden? 


\section{Abkürzungsverzeichnis}

Abs.

Absatz

ANOVA

Analysis of Variance

bzw.

beziehungsweise

DGHD

Deutsche Gesellschaft für Hochschuldidaktik

DIN

Deutsche Industrienorm

ERCP

endoskopische retrograde Cholangio-Pankreatikographie

ICC

Intraklassenkorrelationskoeffizient

IMPP

Institut für medizinische und pharmazeutische Prüfungsfragen

$\mathrm{KI}$

Konfidenzintervall

NKLM

Nationaler Kompetenzbasierter Lernzielkatalog Medizin

OP

Operation

OSCE

Objective Structured Clinical Examination

S.o.

siehe oben

sog.

so genannter/so genannte/so genanntes

$\mathrm{SP}$

Schauspielpatient/in, alternativ: Simulationspatient/in

SPSS

Statistical Package for the Social Sciences

u.a.

unter anderem

z.B.

zum Beispiel

Z.n.

Zustand nach 


\section{Abbildungsverzeichnis}

Abbildung 1: Auszug aus einer Bewertungscheckliste. 15

Abbildung 2: Mittlere Punktescores mit Standardabweichungen in Abhängigkeit der fünf Bewertungsquellen. 25

Abbildung 3: Beurteilerübereinstimmung je Item auf Basis der Intraklassenkorrelation (ICC, unjustiert, 95\%-KI). 27 


\section{Tabellenverzeichnis}

Tabelle 1: Übersicht über die aufzuklärenden Eingriffe ............................................... 9

Tabelle 2: Graphische Darstellung des Ablaufs eines Kommunikationstrainings .......... 12

Tabelle 3: Cronbachs Alpha für die 3 Bewertungschecklisten (Anzahl der Items je Skala

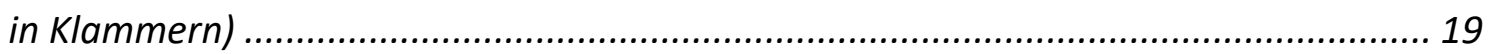

Tabelle 4: Deskriptive Itemkennwerte zur Bewertungscheckliste "Appendektomie“.... 20

Tabelle 5: Deskriptive Itemkennwerte zur Bewertungscheckliste "Cholezystektomie“ . 21

Tabelle 6: Deskriptive Itemkennwerte zur Bewertungscheckliste „Leistenhernie“ ........ 23 


\section{Danksagung}

Hinter jeder gelungenen Doktorarbeit steht neben der Verfasserin bzw. dem Verfasser eine Reihe von Personen, welche zu unterschiedlichen Teilen am Entstehungsprozess mitgewirkt haben. Diesen Menschen möchte ich an dieser Stelle meinen innigsten Dank aussprechen.

Allen voran sei meiner Doktormutter und Betreuerin, Univ.-Prof. Dr. med. Sarah König, MME, gedankt. Sie hat mich vom ersten Kennenlern-Gespräch Ende 2016 an auf Schritt und Tritt begleitet und stand quasi zu jeder Zeit (auch im Urlaub) immer gerne für Rückfragen zur Verfügung. Jede von mir an sie gerichtete E-Mail wurde innerhalb von 24 Stunden beantwortet. Sie war es auch, die sich von Anfang an dafür eingesetzt hat, dass Inhalte der vorliegenden Arbeit in einer themenbezogenen Fachzeitschrift publiziert werden.

Eine Frau der ersten Stunde war auch Dipl.-Psych. Sonia Sippel, welche sowohl bei der Vorbereitung und Durchführung des Kommunikationstrainings beteiligt, als auch bei den komplexen Berechnungen und Auswertungen der Studienergebnisse mithilfe der Software SPSS 24 federführend war. Ihr sei ein herzliches Dankeschön gesagt.

Für all die kleinen, unscheinbaren Dienste, welche von organisatorischer Seite oftmals von Nöten waren, sei an dieser Stelle der Teamassistenz des Instituts, Frau Kerstin Kasseckert gedankt. Sie hat sich jedes Mal prompt um die Planung von Terminen für Besprechungen und Treffen gekümmert und Vieles im Hintergrund vorbereitet, sei es die eine oder andere Kopie, sei es das Vorbereiten der Räumlichkeiten für das Kommunikationstraining.

Wie aus der vorliegenden Arbeit deutlich hervorgeht, war das Projekt Kommunikationstraining keine One-Man-Show. Vielmehr haben mich zwei Kommilitoninnen und ein Kommilitone während der Unterrichtseinheiten als Tutorinnen/Tutor unterstützt. Namentlich seien deshalb Larissa Arras, Johanna Schlei und Christoph Böddeker genannt. 
Auch meiner Tante Sandra Augustin gebührt an dieser Stelle ein großer Dank. Als freischaffende Grafikdesignerin versteht sie die Erstellung von Gliederungen und Layouts mittels Microsoft Word bis ins kleinste Detail. Mit Ihrer Hilfe hat die vorliegende Arbeit ihre aktuelle Form erhalten.

Nicht zuletzt möchte ich von ganzem Herzen meiner Ehefrau Elisabeth Kahr danken. Sie war es, die mir während der unzähligen Stunden der Vorbereitung, Auswertung und Erstellung dieses Dissertationsprojektes Gesellschaft geleistet und mich zum Durchhalten angespornt hat, während ich kurz davor war, die Geduld zu verlieren.

Noch ein Letztes sei an dieser Stelle angeführt: Als Meilenstein auf dem Weg zur medizinischen Promotion sollten Teile der vorliegenden Forschungsarbeit im Rahmen einer hochschuldidaktischen Fachtagung vorgestellt werden. Dies geschah auf der in Räumlichkeiten des Karlsruher Institutes für Technologie stattfindenden 47. Jahrestagung der Deutschen Gesellschaft für Hochschuldidaktik. In der Zeit von 27. Februar bis 2. März 2018 bot sich für den Autor die Möglichkeit einer Reise nach Karlsruhe, um sich dort in Vorlesungen und Workshops fortzubilden, mit Kolleginnen und Kollegen anderer didaktischer Fachrichtungen auszutauschen und an einem Nachmittag Teilaspekte der Studie auf Basis eines Posters im Format DIN A1 vorzustellen und interessierten Besucherinnen und Besuchern Rede und Antwort zu stehen. Dies war eine willkommene Vorbereitung auf die weiteren Schritte der Ergebnisaufbereitung sowie eine Präsentation während des Doktorandenkolloquiums der Würzburger Institute für Medizinische Lehre und Ausbildungsforschung sowie Allgemeinmedizin. Für die Anregung und Ermöglichung der Teilnahme an der DGHD-Jahrestagung 2018 möchte ich mich auf diesem Wege nochmal sehr herzlich bei Frau Prof. Dr. med. Sarah König bedanken. 


\section{Eigene Veröffentlichung und Kongressteilnahme}

- Teilergebnisse der vorliegenden Arbeit wurden im Dezember 2019 im Zentralblatt für Chirurgie veröffentlicht. Der Erstautor ist Philipp Kahr, die Ko-Autoren sind Sonia Sippel und Sarah König. Vollständige Zitierung: Kahr, P., Sippel, S., \& König, S. (2019). Strukturiertes Feedback im Kommunikationstraining zur präoperativen Aufklärung: unterschiedliche Bewertungsquellen nutzbar machen. Zentralblatt für Chirurgie-Zeitschrift für Allgemeine, Viszeral-, Thorax-und Gefäßchirurgie, 144(06), 587-596.

- Teilnahme und Posterpräsentation im Rahmen der 47. Jahrestagung der DGHD 2018 in Karlsruhe 


\section{Ehrenwörtliche Erklärung}

Hiermit erkläre ich, die Dissertation mit dem Titel „Strukturiertes Feedback im Kommunikationstraining zur präoperativen Aufklärung: unterschiedliche Bewertungsquellen nutzbar machen“ eigenständig angefertigt und keine anderen als die von mir angegebenen Quellen und Hilfsmittel verwendet zu haben.

Datum, Ort

Unterschrift 